\title{
Canonicity for Cubical Type Theory
}

\author{
Simon Huber ${ }^{1}$ (DD
}

Received: 28 May 2018 / Accepted: 31 May 2018 / Published online: 13 June 2018

(C) The Author(s) 2018

\begin{abstract}
Cubical type theory is an extension of Martin-Löf type theory recently proposed by Cohen, Coquand, Mörtberg, and the author which allows for direct manipulation of $n$ dimensional cubes and where Voevodsky's Univalence Axiom is provable. In this paper we prove canonicity for cubical type theory: any natural number in a context build from only name variables is judgmentally equal to a numeral. To achieve this we formulate a typed and deterministic operational semantics and employ a computability argument adapted to a presheaf-like setting.
\end{abstract}

Keywords Cubical type theory $\cdot$ Dependent type theory $\cdot$ Canonicity

\section{Introduction}

Cubical type theory as presented in [7] is a dependent type theory which allows one to directly argue about $n$-dimensional cubes, and in which function extensionality and Voevodsky's Univalence Axiom [15] are provable. Cubical type theory is inspired by a constructive model of dependent type theory in cubical sets [7] and a previous variation thereof $[6,10]$. One of its important ingredients is that expressions can depend on names to be thought of as ranging over a formal unit interval $\mathbb{I}$.

Even though the consistency of the calculus already follows from its model in cubical sets, desired - and expected — properties like normalization and decidability of type checking are not yet established. This note presents a first step in this direction by proving canonicity for natural numbers in the following form: given a context $I$ of the form $i_{1}: \mathbb{I}, \ldots, i_{k}: \mathbb{I}, k \geq 0$, and a derivation of $I \vdash u: \mathrm{N}$, there is a unique $n \in \mathbb{N}$ with $I \vdash u=\mathrm{S}^{n} 0: \mathrm{N}$. This $n$ can moreover be effectively calculated. Canonicity in this form also gives an alternative proof of the consistency of cubical type theory (see Corollary 2).

Simon Huber

simon.huber@cse.gu.se

1 Department of Computer Science and Engineering, University of Gothenburg, 41296 Göteborg, Sweden 
The main idea to prove canonicity is as follows. First, we devise an operational semantics given by a typed and deterministic weak-head reduction included in the judgmental equality of cubical type theory. This is given for general contexts although we later on will only use it on terms whose only free variables are name variables, i.e., variables of type $\mathbb{I}$. One result we obtain is that our reduction relation is "complete" in the sense that any term in a name context whose type is the natural numbers can be reduced to one in weak-head normal form (so to zero or a successor). Second, we will follow Tait's computability method $[12,13]$ and devise computability predicates on typed expressions in name contexts and corresponding computability relations (to interpret judgmental equality). These computability predicates are indexed by the list of free name variables of the involved expressions and should be such that substitution induces a cubical set structure on them. This poses a major difficulty given that the reduction relation is in general not closed under name substitutions. A solution is to require for computability that reduction should behave "coherently" with substitution: simplified, reducing an expression and then substituting should be related, by the computability relation, to first substituting and then reducing. A similar condition appeared independently in the Computational Higher Type Theory of Angiuli et al. [4,5] and Angiuli and Harper [3] who work in an untyped setting; they achieve similar results but for a theory not encompassing the Univalence Axiom.

In a way, our technique can be considered as a presheaf extension of the computability argument given in $[1,2]$; the latter being an adaption of the former using a typed reduction relation instead. A similar extension of this technique has been used to show the independence of Markov's principle in type theory [8].

The rest of the paper is organized as follows. In Sect. 2 we introduce the typed reduction relation. Section 3 defines the computability predicates and relations and shows their important properties. In Sect. 4 we show that cubical type theory is sound w.r.t. the computability predicates; this entails canonicity. Section 5 sketches how to adapt the computability argument for the system extended with the circle and propositional truncation, and we deduce an existence property for existentials defined as truncated $\Sigma$-types. We conclude by summarizing and listing further work in the last section. We assume that the reader is familiar with cubical type theory as given in [7]. The present paper is part of the author's $\mathrm{PhD}$ thesis [11].

\section{Reduction}

In this section we give an operational semantics for cubical type theory in the form of a typed and deterministic weak-head reduction. Below we will introduce the relations $\Gamma \vdash A \succ B$ and $\Gamma \vdash u \succ v: A$. These relations are deterministic in the following sense: if $\Gamma \vdash A \succ B$ and $\Gamma \vdash A \succ C$, then $B$ and $C$ are equal as expressions (i.e., up to $\alpha$-equivalence); and, if $\Gamma \vdash u \succ v: A$ and $\Gamma \vdash u \succ w: B$, then $v$ and $w$ are equal as expressions. Moreover, these relations entail judgmental equality, i.e., if $\Gamma \vdash A \succ B$, then $\Gamma \vdash A=B$, and if $\Gamma \vdash u \succ v: A$, then $\Gamma \vdash u=v: A$.

For a context $\Gamma \vdash$, a $\Gamma$-introduced expression is an expression whose outer form is an introduction, so one of the form

$$
\begin{array}{r}
0, \mathrm{~S} u, \mathrm{~N}, \lambda x: A . u,(x: A) \rightarrow B,(u, v),(x: A) \times B, \mathrm{U},\langle i\rangle u, \text { Path } A u v, \\
{\left[\varphi_{1} t_{1}, \ldots, \varphi_{n} t_{n}\right], \text { glue }[\varphi \mapsto t] a, \text { Glue }[\varphi \mapsto(T, w)] A,}
\end{array}
$$

where we require $\varphi \neq 1 \bmod \Gamma$ (which we from now on write as $\Gamma \vdash \varphi \neq 1: \mathbb{F}$ ) for the latter two cases, and in the case of a system (third to last) we require $\Gamma \vdash \varphi_{1} \vee \cdots \vee \varphi_{n}=1: \mathbb{F}$ 
but $\Gamma \vdash \varphi_{k} \neq 1: \mathbb{F}$ for each $k$. In case $\Gamma$ only contains object and interval variable declarations (and no restrictions $\Delta, \psi$ ) we simply refer to $\Gamma$-introduced as introduced. In such a context, $\Gamma \vdash \varphi=\psi: \mathbb{F}$ iff $\varphi=\psi$ as elements of the face lattice $\mathbb{F}$; since $\mathbb{F}$ satisfies the disjunction property, i.e.,

$$
\varphi \vee \psi=1 \Rightarrow \varphi=1 \quad \text { or } \quad \psi=1
$$

a system as above will never be introduced in such a context without restrictions. We call an expression non-introduced if it is not introduced and abbreviate this as "n.i." (often this is referred to as neutral or non-canonical). A $\Gamma$-introduced expression is normal w.r.t. $\Gamma \vdash \cdot \succ$. and $\Gamma \vdash \cdot \succ \cdot: A$.

We will now give the definition of the reduction relation starting with the rules concerning basic type theory.

$$
\begin{aligned}
& \frac{\Gamma \vdash u \succ v: A \quad \Gamma \vdash A=B}{\Gamma \vdash u \succ v: B} \\
& \frac{\Gamma, x: \mathrm{N} \vdash C \quad \Gamma \vdash z: C(x / 0) \quad \Gamma \vdash s:(x: \mathrm{N}) \rightarrow C \rightarrow C(x / \mathrm{S} x)}{\Gamma \vdash \operatorname{natrec} 0 z s \succ z: C(x / 0)} \\
& \Gamma \vdash t: \mathrm{N} \\
& \frac{\Gamma, x: \mathrm{N} \vdash C \quad \Gamma \vdash z: C(x / 0) \quad \Gamma \vdash s:(x: \mathrm{N}) \rightarrow C \rightarrow C(x / \mathrm{S} x)}{\Gamma \vdash \operatorname{natrec}(\mathrm{S} t) z s \succ s t(\text { natrec } t z s): C(x / \mathrm{S} t)} \\
& \Gamma \vdash t \succ t^{\prime}: \mathrm{N} \\
& \frac{\Gamma, x: \mathrm{N} \vdash C \quad \Gamma \vdash z: C(x / 0) \quad \Gamma \vdash s:(x: \mathrm{N}) \rightarrow C \rightarrow C(x / \mathrm{S} x)}{\Gamma \vdash \operatorname{natrec} t z s \succ \operatorname{natrec} t^{\prime} z s: C\left(x / t^{\prime}\right)} \\
& \frac{\Gamma, x: A \vdash t: B \quad \Gamma \vdash u: A}{\Gamma \vdash(\lambda x: A . t) u \succ t(x / u): B(x / u)} \quad \frac{\Gamma \vdash t \succ t^{\prime}:(x: A) \rightarrow B \quad \Gamma \vdash u: A}{\Gamma \vdash t u \succ t^{\prime} u: B(x / u)} \\
& \frac{\Gamma, x: A \vdash B \quad \Gamma \vdash u: A \quad \Gamma \vdash v: B(x / u)}{\Gamma \vdash(u, v) .1 \succ u: A} \quad \frac{\Gamma \vdash t \succ t^{\prime}:(x: A) \times B}{\Gamma \vdash t .1 \succ t^{\prime} .1: A} \\
& \frac{\Gamma, x: A \vdash B \quad \Gamma \vdash u: A \quad \Gamma \vdash v: B(x / u)}{\Gamma \vdash(u, v) .2 \succ v: B(x / u)} \quad \frac{\Gamma \vdash t \succ t^{\prime}:(x: A) \times B}{\Gamma \vdash t .2 \succ t^{\prime} .2: B\left(x / t^{\prime} .1\right)}
\end{aligned}
$$

Note, natrec $t z s$ is not considered as an application (opposed to the presentation in [7]); also the order of the arguments is different to have the main premise as first argument.

Next, we give the reduction rules for Path-types. Note, that like for $\Pi$-types, there is no $\eta$-reduction or expansion, and also there is no reduction for the end-points of a path.

$$
\frac{\Gamma \vdash A \quad \Gamma, i: \mathbb{I} \vdash t: A \quad \Gamma \vdash r: \mathbb{I}}{\Gamma \vdash(\langle i\rangle t) r \succ t(i / r): A} \quad \frac{\Gamma \vdash t \succ t^{\prime}: \text { Path } A \text { u } \quad \Gamma \vdash r: \mathbb{I}}{\Gamma \vdash t r \succ t^{\prime} r: A}
$$

The next rules concern reductions for Glue. 


$$
\begin{gathered}
\frac{\Gamma \vdash A \quad \Gamma, \varphi \vdash T \quad \Gamma, \varphi \vdash w: \text { Equiv } T \quad \Gamma \vdash \varphi=1: \mathbb{F}}{\Gamma \vdash \operatorname{Glue}[\varphi \mapsto(T, w)] A \succ T} \\
\frac{\Gamma, \varphi \vdash w: \text { Equiv } T A \quad \Gamma, \varphi \vdash t: T \quad \Gamma \vdash a: A[\varphi \mapsto w .1 t] \quad \Gamma \vdash \varphi=1: \mathbb{F}}{\Gamma \vdash \operatorname{glue}[\varphi \mapsto t] a \succ t: T} \\
\frac{\Gamma, \varphi \vdash w: \text { Equiv } T A \quad \Gamma, \varphi \vdash t: T \quad \Gamma \vdash a: A[\varphi \mapsto w .1 t] \quad \Gamma \vdash \varphi \neq 1: \mathbb{F}}{\Gamma \vdash \text { unglue }[\varphi \mapsto w](\text { glue }[\varphi \mapsto t] a) \succ a: A} \\
\frac{\Gamma, \varphi \vdash w: \text { Equiv } T A \quad \Gamma \vdash u: \text { Glue }[\varphi \mapsto(T, w)] A \quad \Gamma \vdash \varphi=1: \mathbb{F}}{\Gamma \vdash \text { unglue }[\varphi \mapsto w] u \succ w .1 u: A} \\
\frac{\Gamma \vdash u \succ u^{\prime}: \text { Glue }[\varphi \mapsto(T, w)] A \quad \Gamma \vdash \varphi \neq 1: \mathbb{F}}{\Gamma \vdash \text { unglue }[\varphi \mapsto w] u \succ \text { unglue }[\varphi \mapsto w] u^{\prime}: A}
\end{gathered}
$$

Note that in [7] the annotation $[\varphi \mapsto w]$ of unglue was left implicit. The rules for systems are given by:

$$
\begin{gathered}
\Gamma \vdash \varphi_{1} \vee \cdots \vee \varphi_{n}=1: \mathbb{F} \quad \Gamma, \varphi_{i} \vdash A_{i}(1 \leq i \leq n) \\
\frac{\Gamma, \varphi_{i} \wedge \varphi_{j} \vdash A_{i}=A_{j}(1 \leq i, j \leq n) \quad k \text { minimal with } \Gamma \vdash \varphi_{k}=1: \mathbb{F}}{\Gamma \vdash\left[\varphi_{1} A_{1}, \ldots, \varphi_{n} A_{n}\right] \succ A_{k}} \\
\frac{1 \vdash \varphi_{1} \vee \cdots \vee \varphi_{n}=1: \mathbb{F} \quad \Gamma \vdash A \quad \Gamma, \varphi_{i} \vdash t_{i}: A(1 \leq i \leq n)}{\Gamma, \varphi_{i} \wedge \varphi_{j} \vdash t_{i}=t_{j}: A(1 \leq i, j \leq n) \quad k \text { minimal with } \Gamma \vdash \varphi_{k}=1: \mathbb{F}} \\
\hline \Gamma \vdash\left[\varphi_{1} t_{1}, \ldots, \varphi_{n} t_{n}\right] \succ t_{k}: A
\end{gathered}
$$

The reduction rules for the universe are:

$$
\begin{aligned}
& \frac{\Gamma \vdash A \succ B: U}{\Gamma \vdash A \succ B} \\
& \frac{\Gamma \vdash A: \bigcup \quad \Gamma, \varphi \vdash T: \bigcup \quad \Gamma, \varphi \vdash w: \text { Equiv } T A \quad \Gamma \vdash \varphi=1: \mathbb{F}}{\Gamma \vdash \text { Glue }[\varphi \mapsto(T, w)] A \succ T: U}
\end{aligned}
$$

Finally, the reduction rules for compositions are given as follows.

$$
\begin{gathered}
\frac{\Gamma, i: \mathbb{I} \vdash A \succ B \quad \Gamma \vdash \varphi: \mathbb{F} \quad \Gamma, \varphi, i: \mathbb{I} \vdash u: A \quad \Gamma \vdash u_{0}: A(i 0)[\varphi \mapsto u(i 0)]}{\Gamma \vdash \operatorname{comp}^{i} A[\varphi \mapsto u] u_{0} \succ \operatorname{comp}^{i} B[\varphi \mapsto u] u_{0}: B(i 1)} \\
\frac{\Gamma \vdash \varphi: \mathbb{F} \quad \Gamma, \varphi, i: \mathbb{I} \vdash u: \mathrm{N} \quad \Gamma, \varphi, i: \mathbb{I} \vdash u=0: \mathrm{N}}{\Gamma \vdash \operatorname{comp}^{i} \mathrm{~N}[\varphi \mapsto u] 0 \succ 0: \mathrm{N}} \\
\Gamma \vdash \varphi: \mathbb{F} \quad \Gamma, \varphi, i: \mathbb{I} \vdash u: \mathrm{N} \quad \Gamma, \varphi, i: \mathbb{I} \vdash w: \mathrm{N} \\
\frac{\Gamma, \varphi, i: \mathbb{I} \vdash u=\mathrm{S} w: \mathrm{N} \quad \Gamma \vdash u_{0}: \mathrm{N} \quad \Gamma, \varphi \vdash u(i 0)=\mathrm{S} u_{0}: \mathrm{N}}{\Gamma \vdash \operatorname{comp}^{i} \mathrm{~N}[\varphi \mapsto u]\left(\mathrm{S} u_{0}\right) \succ \mathrm{S}\left(\operatorname{comp}^{i} \mathrm{~N}[\varphi \mapsto \operatorname{pred} u] u_{0}\right): \mathrm{N}}
\end{gathered}
$$


Here pred is the usual predecessor function defined using natrec. ${ }^{1}$

$$
\begin{aligned}
& \frac{\Gamma \vdash \varphi: \mathbb{F} \quad \Gamma, \varphi, i: \mathbb{I} \vdash u: \mathrm{N} \quad \Gamma \vdash u_{0}: \mathrm{N}[\varphi \mapsto u(i 0)] \quad \Gamma \vdash u_{0} \succ v_{0}: \mathrm{N}}{\Gamma \vdash \operatorname{comp}^{i} \mathrm{~N}[\varphi \mapsto u] u_{0} \succ \operatorname{comp}^{i} \mathrm{~N}[\varphi \mapsto u] v_{0}: \mathrm{N}} \\
& \Gamma \vdash \varphi: \mathbb{F} \quad \Gamma, i: \mathbb{I} \vdash A \quad \Gamma, i: \mathbb{I}, x: A \vdash B \\
& \Gamma, \varphi, i: \mathbb{I} \vdash u:(x: A) \rightarrow B \quad \Gamma \vdash u_{0}:((x: A) \rightarrow B)(i 0)[\varphi \mapsto u(i 0)] \\
& \Gamma \vdash \operatorname{comp}^{i}((x: A) \rightarrow B)[\varphi \mapsto u] u_{0} \succ \\
& \lambda y: A(i 1) \cdot \operatorname{comp}^{i} B(x / \bar{y})[\varphi \mapsto u \bar{y}]\left(u_{0} \bar{y}(i 0)\right):(x: A(i 1)) \rightarrow B(i 1) \\
& \text { where } y^{\prime}=\text { fill }^{i} A(i / 1-i)[] y \text { and } \bar{y}=y^{\prime}(i / 1-i) \\
& \Gamma \vdash \varphi: \mathbb{F} \quad \Gamma, i: \mathbb{I} \vdash A \quad \Gamma, i: \mathbb{I}, x: A \vdash B \\
& \frac{\Gamma, \varphi, i: \mathbb{I} \vdash u:(x: A) \times B \quad \Gamma \vdash u_{0}:((x: A) \times B)(i 0)[\varphi \mapsto u(i 0)]}{\Gamma \vdash \operatorname{comp}^{i}((x: A) \times B)[\varphi \mapsto u] u_{0} \succ} \\
& \left(v(i 1), \operatorname{comp}^{i} B(x / v)[\varphi \mapsto u .2]\left(u_{0} .2\right)\right):(x: A(i 1)) \times B(i 1) \\
& \text { where } v=\text { fill }^{i} A[\varphi \mapsto u .1]\left(u_{0} .1\right) \\
& \Gamma \vdash \varphi: \mathbb{F} \quad \Gamma, i: \mathbb{I} \vdash A \quad \Gamma, i: \mathbb{I} \vdash v: A \quad \Gamma, i: \mathbb{I} \vdash w: A \\
& \Gamma, \varphi, i: \mathbb{I} \vdash u \text { : Path } A v w \quad \Gamma \vdash u_{0} \text { : Path } A(i 0) v(i 0) w(i 0)[\varphi \mapsto u(i 0)] \\
& \left.\Gamma \vdash \operatorname{comp}^{i} \text { (Path } A v w\right)[\varphi \mapsto u] u_{0} \succ \\
& \langle j\rangle \operatorname{comp}^{i} A[(j=0) \mapsto v,(j=1) \mapsto w, \varphi \mapsto u j]\left(u_{0} j\right): \text { Path } A(i 1) v(i 1) w(i 1) \\
& \Gamma, i: \mathbb{I} \vdash A \quad \Gamma, i: \mathbb{I} \vdash \varphi: \mathbb{F} \quad \Gamma, i: \mathbb{I} \vdash \varphi \neq 1: \mathbb{F} \quad \Gamma, i: \mathbb{I}, \varphi \vdash T \\
& \Gamma, i: \mathbb{I}, \varphi \vdash w: \text { Equiv } T A \quad \Gamma \vdash \psi: \mathbb{F} \quad \Gamma, \psi, i: \mathbb{I} \vdash u: \text { Glue }[\varphi \mapsto(T, w)] A \\
& \Gamma \vdash u_{0}:(\text { Glue }[\varphi \mapsto(T, w)] A)(i 0)[\psi \mapsto u(i 0)] \\
& \Gamma \vdash \operatorname{comp}^{i}(\text { Glue }[\varphi \mapsto(T, w)] A)[\psi \mapsto u] u_{0} \succ \\
& \text { glue }\left[\varphi(i 1) \mapsto t_{1}\right] a_{1}:(\text { Glue }[\varphi \mapsto(T, w)] A)(i 1)
\end{aligned}
$$

Here $a_{1}$ and $t_{1}$ are defined like in [7], i.e., given by

$$
\begin{aligned}
a & =\text { unglue }[\varphi \mapsto w] u & & \Gamma, i: \mathbb{I}, \psi \\
a_{0} & =\text { unglue }[\varphi(i 0) \mapsto w(i 0)] u_{0} & & \Gamma \\
\delta & =\forall i . \varphi & & \Gamma \\
a_{1}^{\prime} & =\operatorname{comp}^{i} A[\psi \mapsto a] a_{0} & & \Gamma \\
t_{1}^{\prime} & =\operatorname{comp}^{i} T[\psi \mapsto u] u_{0} & & \Gamma, \delta \\
\omega & =\operatorname{pres}^{i} w[\psi \mapsto u] u_{0} & & \Gamma, \delta \\
\left(t_{1}, \alpha\right) & =\operatorname{equiv} w(i 1)\left[\delta \mapsto\left(t_{1}^{\prime}, \omega\right), \psi \mapsto\left(u(i 1),\langle j\rangle a_{1}^{\prime}\right)\right] a_{1}^{\prime} & & \Gamma, \varphi(i 1) \\
a_{1} & =\operatorname{comp}^{j} A(i 1)[\varphi(i 1) \mapsto \alpha j, \psi \mapsto a(i 1)] a_{1}^{\prime} & & \Gamma
\end{aligned}
$$

where we indicated the intended context on the right.

$$
\frac{\Gamma \vdash \varphi: \mathbb{F} \quad \Gamma, \varphi, i: \mathbb{I} \vdash u: \cup \quad \Gamma \vdash u_{0}: \mathrm{U}[\varphi \mapsto u(i 0)]}{\Gamma \vdash \operatorname{comp}^{i} \mathrm{U}[\varphi \mapsto u] u_{0} \succ \text { Glue }\left[\varphi \mapsto\left(u(i 1), \text { equiv }^{i} u(i / 1-i)\right)\right] u_{0}: \bigcup}
$$

\footnotetext{
1 This trick allows us that we never have to reduce in the system of a composition when defining composition for natural numbers, which also gives that reduction over $\Gamma$ never refers to reduction in a restricted context $\Gamma, \varphi$ (given that $\Gamma$ is not restricted). If we would instead directly require $u$ above to be of the form $\mathrm{S} u^{\prime}$, we would have to explain reductions for systems like $\left[(i=0)(\mathrm{S} t),(i=1)\left(\mathrm{S} t^{\prime}\right)\right]$ and more generally how reduction and systems interact.
} 
Here equiv ${ }^{i}$ is defined as in [7]. This concludes the definition of the reduction relation.

For $\Gamma \vdash A$ we write $A !_{\Gamma}$ if there is $B$ such that $\Gamma \vdash A \succ B$; in this case $B$ is uniquely determined by $A$ and we denote $B$ by $A \downarrow_{\Gamma}$; if $A$ is normal we set $A \downarrow_{\Gamma}$ to be $A$. Similarly for $\Gamma \vdash u: A, u !_{\Gamma}^{A}$ and $u \downarrow{ }_{\Gamma}^{A}$. Note that if a term or type has a reduct it is non-introduced. We usually drop the subscripts and sometimes also superscripts since they can be inferred.

From now on we will mainly consider contexts $I, J, K, \ldots$ only built from dimension name declarations; so such a context is of the form $i_{1}: \mathbb{I}, \ldots, i_{n}: \mathbb{I}$ for $n \geq 0$. We sometimes write $I, i$ for $I, i: \mathbb{I}$. Substitutions between such contexts will be denoted by $f, g, h, \ldots$. The resulting category with such name contexts $I$ as objects and substitutions $f: J \rightarrow I$ is reminiscent of the category of cubes as defined in [7, Section 8.1] with the difference that the names in a contexts $I$ are ordered and not sets. This difference is not crucial for the definition of computability predicates in the next section but it simplifies notations. (Note that if $I^{\prime}$ is a permutation of $I$, then the substitution assigning to each name in $I$ itself is an isomorphism $I^{\prime} \rightarrow I$.) We write $r \in \mathbb{I}(I)$ if $I \vdash r: \mathbb{I}$, and $\varphi \in \mathbb{F}(I)$ if $I \vdash \varphi: \mathbb{F}$.

Note that in general reductions $I \vdash A \succ B$ or $I \vdash u \succ v: A$ are not closed under substitutions $f: J \rightarrow I$. For example, if $u$ is a system $\left[(i=0) u_{1}, 1 u_{2}\right]$, then $i \vdash u \succ u_{2}: A$ (assuming everything is well typed), but $\vdash u(i 0) \succ u_{1}(i 0): A(i 0)$ and $u_{1}, u_{2}$ might be chosen that $u_{1}(i 0)$ and $u_{2}(i 0)$ are judgmentally equal but not syntactically (and even normal by considering two $\lambda$-abstractions where the body is not syntactically but judgmentally equal). Another example is when $u$ is unglue $[\varphi \mapsto w]$ (glue $[\varphi \mapsto t] a)$ with $\varphi \neq 1$ and with $f: J \rightarrow I$ such that $\varphi f=1$; then $u$ reduces to $a$, but $u f$ reduces to $w f .1$ (glue $[\varphi f \mapsto$ $t f] a f$ ) which is in general not syntactically equal to $a f$.

We write $I \vdash A \succ_{\mathrm{s}} B$ and $I \vdash u \succ_{\mathrm{s}} v: A$ if the respective reduction is closed under name substitutions. That is, $I \vdash A \succ_{\mathrm{s}} B$ whenever $J \vdash A f \succ B f$ for all $f: J \rightarrow I$. Note that in the above definition, all the rules which do not have a premise with a negated equation in $\mathbb{F}$ and which do not have a premise referring to another reduction are closed under substitution.

\section{Computability Predicates}

In this section we define computability predicates and establish the properties we need for the proof of Soundness in the next section. We will define when a type is computable or forced, written $I \Vdash_{\ell} A$, when two types are forced equal, $I \Vdash_{\ell} A=B$, when an element is computable or forced, $I \Vdash_{\ell} u: A$, and when two elements are forced equal, $I \Vdash_{\ell} u=v: A$. Here $\ell$ is the level which is either 0 or 1 , the former indicating smallness.

The definition is given as follows: by main recursion on $\ell$ (that is, we define " $\vdash_{0}$ " before " $\vdash_{1}$ ") we define by induction-recursion [9]

$$
\begin{aligned}
& I \Vdash_{\ell} A \\
& I \Vdash_{\ell} A=B \\
& I \Vdash_{\ell} u: A \\
& I \Vdash_{\ell} u=v: A
\end{aligned}
$$

by recursion on $I \Vdash_{\ell} A$

by recursion on $I \Vdash_{\ell} A$

where the former two are mutually defined by induction, and the latter two mutually by recursion on the derivation of $I \Vdash_{\ell} A$. Formally, $I \Vdash_{\ell} A$ and $I \Vdash_{\ell} A=B$ are witnessed by derivations for which we don't introduce notations since the definitions of $I \Vdash_{\ell} u: A$ and $I \Vdash_{\ell} u=v: A$ don't depend on the derivation of $I \Vdash_{\ell} A$. Each such derivation has a height as an ordinal, and often we will employ induction not only on the structure of such a derivation but on its height. 
Note that the arguments and definitions can be adapted to a hierarchy of universes by allowing $\ell$ to range over a (strict) well-founded poset.

We write $I \Vdash_{\ell} A \doteqdot B$ for the conjunction of $I \Vdash_{\ell} A, I \Vdash_{\ell} B$, and $I \Vdash_{\ell} A=B$. For $\varphi \in \mathbb{F}(I)$ we write $f: J \rightarrow I, \varphi$ for $f: J \rightarrow I$ with $\varphi f=1$; furthermore we write

$$
\begin{array}{lll}
I, \varphi \Vdash_{\ell} A & \text { for } & \forall f: J \rightarrow I, \varphi\left(J \Vdash_{\ell} A f\right) \& I, \varphi \vdash A, \\
I, \varphi \Vdash_{\ell} A=B & \text { for } & \forall f: J \rightarrow I, \varphi\left(J \Vdash_{\ell} A f=B f\right) \& I, \varphi \vdash A=B, \\
I, \varphi \Vdash_{\ell} u: A & \text { for } & \forall f: J \rightarrow I, \varphi\left(J \Vdash_{\ell} u f: A f\right) \& I, \varphi \vdash u: A, \\
I, \varphi \Vdash_{\ell} u=v: A & \text { for } & \forall f: J \rightarrow I, \varphi\left(J \Vdash_{\ell} u f=v f: A f\right) \& I, \varphi \vdash u=v: A
\end{array}
$$

Where the last two abbreviations need suitable premises to make sense. Note that $I, 1 \Vdash_{\ell} A$ is a priori stronger than $I \Vdash_{\ell} A$; that these notions are equivalent follows from the Monotonicity Lemma below. Moreover, the definition is such that $I \vdash \mathscr{J}$ whenever $I \Vdash_{\ell} \mathscr{J}$ (where $\mathscr{J}$ is any judgment form); it is shown in Remark 4 that the condition $I, \varphi \vdash \mathscr{J}$ in the definition of $I, \varphi \Vdash_{\ell} \mathscr{J}$ is actually not needed and follows from the other.

$I \Vdash_{\ell} A$ assuming $I \vdash A$ (i.e., the rules below all have a suppressed premise $I \vdash A$ ).

$$
\overline{I \Vdash \ell}^{\mathrm{N}-\mathrm{C}}
$$

$$
\begin{gathered}
\begin{array}{c}
I, 1 \Vdash_{\ell} A \quad I, x: A \vdash B \quad \forall f: J \rightarrow I \forall u\left(J \Vdash_{\ell} u: A f \Rightarrow J \Vdash_{\ell} B(f, x / u)\right) \\
\forall f: J \rightarrow I \forall u, v\left(J \Vdash_{\ell} u=v: A f \Rightarrow J \Vdash_{\ell} B(f, x / u) \doteqdot B(f, x / v)\right)
\end{array} \\
I \Vdash_{\ell}(x: A) \rightarrow B
\end{gathered}
$$

$$
\begin{gathered}
\begin{array}{c}
I, 1 \Vdash_{\ell} A \quad I, x: A \vdash B \quad \forall f: J \rightarrow I \forall u\left(J \Vdash_{\ell} u: A f \Rightarrow J \Vdash_{\ell} B(f, x / u)\right) \\
\forall f: J \rightarrow I \forall u, v\left(J \Vdash_{\ell} u=v: A f \Rightarrow J \Vdash_{\ell} B(f, x / u) \doteqdot B(f, x / v)\right)
\end{array} \\
I \Vdash_{\ell}(x: A) \times B
\end{gathered}
$$

$$
\frac{I, 1 \Vdash_{\ell} A \quad I \Vdash_{\ell} a_{0}: A \quad I \Vdash_{\ell} a_{1}: A}{I \Vdash_{\ell} \text { Path } A a_{0} a_{1}} \text { PA-C }
$$

$$
\begin{gathered}
1 \neq \varphi \in \mathbb{F}(I) \quad I, 1 \Vdash_{\ell} A \\
I, \varphi \Vdash_{\ell} \text { Equiv } T A \quad I, \varphi \Vdash_{\ell} w: \text { Equiv } T A \quad I, \varphi \Vdash_{\ell} \text { Glue }[\varphi \mapsto(T, w)] A \\
I \Vdash_{\ell} \text { Glue }[\varphi \mapsto(T, w)] A
\end{gathered} \text { GL-C }
$$

$$
\begin{aligned}
& A \text { n.i. } \quad \forall f: J \rightarrow I\left(A f ! \& J \Vdash_{\ell} A f \downarrow\right) \\
& \frac{}{I \Vdash_{1} \mathrm{U}} \mathrm{U}-\mathrm{C} \quad \frac{\forall f: J \rightarrow I \forall g: K \rightarrow J\left(K \Vdash_{\ell} A f \downarrow g=A f g \downarrow\right)}{I \Vdash_{\ell} A} \text { NI-C }
\end{aligned}
$$

Note, that the rule GL-C above is not circular, as for any $f: J \rightarrow I, \varphi$ we have $\varphi f=1$ and so (Glue $[\varphi \mapsto(T, w)] A) f$ is non-introduced.

$I \Vdash_{\ell} A=B$ assuming $I \Vdash_{\ell} A, I \Vdash_{\ell} B$, and $I \vdash A=B$ (i.e., each rule below has the suppressed premises $I \Vdash_{\ell} A, I \Vdash_{\ell} B$, and $I \vdash A=B$ ). 


$$
\overline{I \Vdash_{\ell} \mathrm{N}=\mathrm{N}} \mathrm{N}-\mathrm{E}
$$

$I, 1 \Vdash_{\ell} A=A^{\prime}$

$\frac{I, x: A \vdash B=B^{\prime} \quad \forall f: J \rightarrow I \forall u\left(J \Vdash_{\ell} u: A f \Rightarrow J \Vdash_{\ell} B(f, x / u)=B^{\prime}(f, x / u)\right)}{I \Vdash_{\ell}(x: A) \rightarrow B=\left(x: A^{\prime}\right) \rightarrow B^{\prime}}$ PI-E

$$
I, 1 \Vdash_{\ell} A=A^{\prime}
$$

$\frac{I, x: A \vdash B=B^{\prime} \quad \forall f: J \rightarrow I \forall u\left(J \Vdash_{\ell} u: A f \Rightarrow J \Vdash_{\ell} B(f, x / u)=B^{\prime}(f, x / u)\right)}{I \Vdash_{\ell}(x: A) \times B=\left(x: A^{\prime}\right) \times B^{\prime}}$ SI-E

$$
\frac{I, 1 \Vdash_{\ell} A=B \quad I \Vdash_{\ell} a_{0}=b_{0}: A \quad I \Vdash_{\ell} a_{1}=b_{1}: A}{I \Vdash_{\ell} \text { Path } A a_{0} a_{1}=\text { Path } B b_{0} b_{1}} \text { PA-E }
$$

$1 \neq \varphi \in \mathbb{F}(I) I, \quad 1 \Vdash_{\ell} A=A^{\prime} \quad I, \varphi \Vdash_{\ell}$ Equiv $T A=$ Equiv $T^{\prime} A^{\prime}$

$I, \varphi \Vdash_{\ell} w=w^{\prime}:$ Equiv $T A \quad I, \varphi \Vdash_{\ell}$ Glue $[\varphi \mapsto(T, w)] A=$ Glue $\left[\varphi \mapsto\left(T^{\prime}, w^{\prime}\right)\right] A^{\prime}$

$$
I \Vdash_{\ell} \text { Glue }[\varphi \mapsto(T, w)] A=\text { Glue }\left[\varphi \mapsto\left(T^{\prime}, w^{\prime}\right)\right] A^{\prime}
$$

$$
\frac{A \text { or } B \text { n.i. } \quad \forall f: J \rightarrow I\left(J \Vdash_{\ell} A f \downarrow=B f \downarrow\right)}{I \Vdash_{1} \mathrm{U}=\mathrm{U}} \mathrm{U}-\mathrm{E} \quad \text { NI-E }
$$

$I \Vdash_{\ell} u: A$ by induction on $I \Vdash_{\ell} A$ assuming $I \vdash u: A$. We distinguish cases on the derivation of $I \Vdash_{\ell} A$.

Case N-C

$$
u \text { n.i. } \quad \forall f: J \rightarrow I\left(u f !^{\mathrm{N}} \& J \Vdash_{\ell} u f \downarrow^{\mathrm{N}}: \mathrm{N}\right)
$$

$\frac{I \Vdash_{\ell} u: \mathrm{N}}{I \Vdash_{\ell} 0: \mathrm{N}} \quad \frac{\forall f: J \rightarrow I \forall g: K \rightarrow J\left(K \Vdash_{\ell} u f \downarrow \Vdash_{\ell} \mathrm{S} u: \mathrm{N}\right.}{I \Vdash_{\ell} u: \mathrm{N}} \quad \frac{u f g \downarrow}{\mathrm{N}: \mathrm{N})}$

Case PI-C

$$
\begin{gathered}
\forall f: J \rightarrow I \forall u\left(J \Vdash_{\ell} u: A f \Rightarrow J \Vdash_{\ell} w f u: B(f, x / u)\right) \\
\forall f: J \rightarrow I \forall u, v\left(J \Vdash_{\ell} u=v: A f \Rightarrow J \Vdash_{\ell} w f u=w f v: B(f, x / u)\right) \\
I \Vdash_{\ell} w:(x: A) \rightarrow B
\end{gathered}
$$

Case SI-C

$$
\frac{I \Vdash_{\ell} u .1: A \quad I \Vdash_{\ell} u .2: B(x / u .1)}{I \Vdash_{\ell} u:(x: A) \times B}
$$

\section{Case PA-C}

$\forall f: J \rightarrow I \forall r \in \mathbb{I}(J)\left(J \Vdash_{\ell}\right.$ ufr $:$ Af $) \quad I \Vdash_{\ell} u 0=a_{0}: A \quad I \Vdash_{\ell} u 1=a_{1}: A$ 
Case GL-C

$$
\begin{gathered}
I, \varphi \Vdash_{\ell} u: \text { Glue }[\varphi \mapsto(T, w)] A \\
\forall f: J \rightarrow I \forall w^{\prime}\left(J, \varphi f \Vdash w^{\prime}=w f: \text { Equiv Tf Af } \Rightarrow\right. \\
\left.J \Vdash \text { unglue }\left[\varphi f \mapsto w^{\prime}\right] u f=\text { unglue }[\varphi f \mapsto w f] u f: A f\right) \\
\hline I \Vdash_{\ell} u: \text { Glue }[\varphi \mapsto(T, w)] A
\end{gathered}
$$

Later we will see that from the premises of GL-C we get $I \Vdash w=w$ : Equiv $T A$, and the second premise above implies in particular $I \Vdash$ unglue $[\varphi \mapsto w] u: A$; the quantification over other possible equivalences is there to ensure invariance for the annotation.

Case U-C

$$
\frac{I \Vdash_{0} A}{I \Vdash_{1} A: \mathrm{U}}
$$

Case NI-C

$$
\frac{\forall f: J \rightarrow I\left(J \Vdash_{\ell} u f: A f \downarrow\right)}{I \Vdash_{\ell} u: A}
$$

$I \Vdash_{\ell} u=v: A$ by induction on $I \Vdash_{\ell} A$ assuming $I \Vdash_{\ell} u: A, I \Vdash_{\ell} v: A$, and $I \vdash u=$

\begin{tabular}{|c|c|c|}
\hline & $I \Vdash_{\ell} u=v: \mathrm{N}$ & $\forall f\left(J \Vdash_{\ell} u f \downarrow^{\mathrm{N}}=v f \downarrow^{\mathrm{N}}: \mathrm{N}\right)$ \\
\hline $\bar{I} \Vdash_{\ell} 0=0: \mathrm{N}$ & $\bar{I} \Vdash_{\ell} \mathrm{S} u=\mathrm{S} v: \mathrm{N}$ & $I \Vdash_{\ell} u=v: \mathrm{N}$ \\
\hline
\end{tabular}
$v: A$. (I.e., each of the rules below has the suppressed premises $I \Vdash_{\ell} u: A, I \Vdash_{\ell} v: A$, and $I \vdash u=v: A$, but they are not arguments to the definition of the predicate. This is subtle since in, e.g., the rule for pairs we only know $I \Vdash_{\ell} v .2: B(x / v .1)$ not $I \Vdash_{\ell} v .2: B(x / u .1)$.) We distinguish cases on the derivation of $I \Vdash_{\ell} A$.

Case N-C

Case PI-C

$$
\frac{\forall f: J \rightarrow I \forall u\left(J \Vdash_{\ell} u: A f \Rightarrow J \Vdash_{\ell} w f u=w^{\prime} f u: B(f, x / u)\right)}{I \Vdash_{\ell} w=w^{\prime}:(x: A) \rightarrow B}
$$

Case SI-C

$$
\frac{I \Vdash_{\ell} u .1=v .1: A \quad I \Vdash_{\ell} u .2=v .2: B(x / u .1)}{I \Vdash_{\ell} u=v:(x: A) \times B}
$$

Case PA-C

$$
\frac{\forall f: J \rightarrow I \forall r \in \mathbb{I}(J)\left(J \Vdash_{\ell} u f r=v f r: A f\right)}{I \Vdash_{\ell} u=v: \text { Path } A a_{0} a_{1}}
$$

Case GL-C

$$
\begin{gathered}
I, \varphi \Vdash_{\ell} u=v: \text { Glue }[\varphi \mapsto(T, w)] A \\
\frac{I, 1 \Vdash_{\ell} \text { unglue }[\varphi \mapsto w] u=\operatorname{unglue}[\varphi \mapsto w] v: A}{I \Vdash_{\ell} u=v: \text { Glue }[\varphi \mapsto(T, w)] A}
\end{gathered}
$$


Case U-C

$$
\frac{I \Vdash_{0} A=B}{I \Vdash_{1} A=B: \bigcup}
$$

Case NI-C

$$
\frac{\forall f: J \rightarrow I\left(J \Vdash_{\ell} u f=v f: A f \downarrow\right)}{I \Vdash_{\ell} u=v: A}
$$

Note that the definition is such that $I \Vdash_{\ell} A=B$ implies $I \Vdash_{\ell} A$ and $I \Vdash_{\ell} B$; and, likewise, $I \Vdash_{\ell} u=v: A$ gives $I \Vdash_{\ell} u: A$ and $I \Vdash_{\ell} v: A$.

Remark 1 1. In the rule NI-E and the rule for $I \Vdash_{\ell} u=v: \mathrm{N}$ in case $u$ or $v$ are nonintroduced we suppressed the premise that the reference to " $\downarrow$ ” is actually well defined; it is easily seen that if $I \Vdash_{\ell} A$, then $A \downarrow$ is well defined, and similarly for $I \Vdash_{\ell} u: \mathrm{N}$, $u \downarrow{ }^{\mathrm{N}}$ is well defined.

2. It follows from the substitution lemma below that $I \Vdash_{\ell} A$ whenever $A$ is non-introduced and $I \vdash A \succ_{\mathrm{s}} B$ with $I \Vdash_{\ell} B$. (Cf. also the Expansion Lemma below.)

3. Note that once we also have proven transitivity, symmetry, and monotonicity, the last premise of NI-C in the definition of $I \Vdash_{\ell} A$ (and similarly in the rule for non-introduced naturals) can be restated as $J \Vdash_{\ell} A f \downarrow=A \downarrow f$ for all $f: J \rightarrow I$.

Lemma 1 The computability predicates are independent of the derivation, i.e., if we have two derivations trees $d_{1}$ and $d_{2}$ of $I \Vdash_{\ell} A$, then

$$
\begin{aligned}
& I \Vdash_{\ell}^{d_{1}} u: A \Leftrightarrow I \Vdash_{\ell}^{d_{2}} u: A, \quad \text { and } \\
& I \Vdash_{\ell}^{d_{1}} u=v: A \Leftrightarrow I \Vdash_{\ell}^{d_{2}} u=v: A
\end{aligned}
$$

where $\Vdash_{\ell}^{d_{i}}$ refers to the predicate induced by $d_{i}$.

Proof By main induction on $\ell$ and a side induction on the derivations $d_{1}$ and $d_{2}$. Since the definition of $I \Vdash_{\ell} A$ is syntax directed both $d_{1}$ and $d_{2}$ are derived by the same rule. The claim thus follows from the IH.

Lemma 2 1. If $I \Vdash_{\ell} A$, then $I \vdash A$ and:

(a) $I \Vdash_{\ell} u: A \Rightarrow I \vdash u: A$,

(b) $I \Vdash_{\ell} u=v: A \Rightarrow I \vdash u=v: A$.

2. If $I \Vdash_{\ell} A=B$, then $I \vdash A=B$.

Lemma 3 1. If $I \Vdash_{0} A$, then:

(a) $I \Vdash_{1} A$

(b) $I \Vdash_{0} u: A \Leftrightarrow I \Vdash_{1} u: A$

(c) $I \Vdash_{0} u=v: A \Leftrightarrow I \Vdash_{1} u=v: A$

2. If $I \Vdash_{0} A=B$, then $I \Vdash_{1} A=B$.

Proof By simultaneous induction on $I \Vdash_{0} A$ and $I \Vdash_{0} A=B$.

We will write $I \Vdash A$ if there is a derivation of $I \Vdash_{\ell} A$ for some $\ell$; etc. Such derivations will be ordered lexicographically, i.e., $I \Vdash_{0} A$ derivations are ordered before $I \Vdash_{1} A$ derivations. 
Lemma 4 1. $I \Vdash_{\ell} A \Rightarrow I \Vdash_{\ell} A=A$

2. $I \Vdash_{\ell} A \& I \Vdash_{\ell} u: A \Rightarrow I \Vdash_{\ell} u=u: A$

Proof Simultaneously, by induction on $\ell$ and side induction on $I \Vdash_{\ell} A$. In the case GL-C, to see (2), note that from the assumption $I \Vdash u: B$ with $B$ being Glue $[\varphi \mapsto(T, w)] A$ we get in particular

$$
I, \varphi \Vdash w=w \text { : Equiv } T A \Rightarrow I \Vdash \text { unglue }[\varphi \mapsto w] u=\text { unglue }[\varphi \mapsto w] u: A \text {. }
$$

But by $\mathrm{IH}$, the premise follows from $I, \varphi \Vdash w$ : Equiv $T A$; moreover, $I, \varphi \Vdash u=u: B$ is immediate by $\mathrm{IH}$, showing $I \Vdash u=u: B$.

Lemma 5 (Monotonicity/Substitution) For $f: J \rightarrow I$ we have

1. $I \Vdash_{\ell} A \Rightarrow J \Vdash_{\ell} A f$,

2. $I \Vdash_{\ell} A=B \Rightarrow J \Vdash_{\ell} A f=B f$,

3. $I \Vdash_{\ell} A \& I \Vdash_{\ell} u: A \Rightarrow J \Vdash_{\ell} u f: A f$,

4. $I \Vdash_{\ell} A \& I \Vdash_{\ell} u=v: A \Rightarrow J \Vdash_{\ell} u f=v f: A f$.

Moreover, the respective heights of the derivations don't increase.

Proof By induction on $\ell$ and side induction on $I \Vdash_{\ell} A$ and $I \Vdash_{\ell} A=B$. The definition of computability predicates and relations is lead such that this proof is immediate. For instance, note for (1) in the case GL-C, i.e.,

$$
\begin{gathered}
1 \neq \varphi \in \mathbb{F}(I) \quad I, 1 \Vdash_{\ell} A \\
I, \varphi \Vdash_{\ell} \text { Equiv } T A \quad I, \varphi \Vdash_{\ell} w: \text { Equiv } T A \quad I, \varphi \Vdash_{\ell} \text { Glue }[\varphi \mapsto(T, w)] A \\
I \Vdash_{\ell} \text { Glue }[\varphi \mapsto(T, w)] A
\end{gathered} \text { GL-C }
$$

we distinguish cases: if $\varphi f=1$, then $J \Vdash_{\ell}$ Glue $[\varphi f \mapsto(T f, w f)] A f$ by the premise $I, \varphi \Vdash_{\ell}$ Glue $[\varphi \mapsto(T, w)] A$; in case $\varphi f \neq 1$ we can use the same rule again.

Lemma 6 1. $I \Vdash A \Rightarrow I \Vdash A \downarrow$

2. $I \Vdash A=B \Rightarrow I \Vdash A \downarrow=B \downarrow$

3. $I \Vdash A \& I \Vdash u: A \Rightarrow I \Vdash u: A \downarrow$

4. $I \Vdash A \& I \Vdash u=v: A \Rightarrow I \Vdash u=v: A \downarrow$

5. $I \Vdash u: \mathrm{N} \Rightarrow I \Vdash u \downarrow: \mathrm{N}$

6. $I \Vdash u=v: \mathrm{N} \Rightarrow I \Vdash u \downarrow=v \downarrow: \mathrm{N}$

Moreover, the respective heights of the derivations don't increase.

Proof (1) By induction on $I \Vdash A$. All cases were $A$ is an introduction are immediate since then $A \downarrow$ is $A$. It only remains the case NI-C:

$A$ n.i.

$\frac{\forall f: J \rightarrow I(A f ! \& J \Vdash A f \downarrow) \quad \forall f: J \rightarrow I \forall g: K \rightarrow J(K \Vdash A f \downarrow g=A f g \downarrow)}{I \Vdash A}$ NI-C

We have $I \Vdash A \downarrow$ as this is one of the premises.

(5) By induction on $I \Vdash u: \mathrm{N}$ similarly to the last paragraph.

(2) By induction on $I \Vdash A=B$. The only case where a reduct may happen is NI-E, in which $I \Vdash A \downarrow=B \downarrow$ is a premise. Similar for (6).

(3) and (4): By induction on $I \Vdash A$, where the only interesting case is NI-C, in which what we have to show holds by definition. 
Lemma 7 1. If $I \Vdash A=B$, then

(a) $I \Vdash u: A \Leftrightarrow I \Vdash u: B$, and

(b) $I \Vdash u=v: A \Leftrightarrow I \Vdash u=v: B$.

2. $I \Vdash A=B \& I \Vdash B=C \Rightarrow I \Vdash A=C$

3. Given $I \Vdash A$ we get

$$
I \Vdash u=v: A \& I \Vdash v=w: A \Rightarrow I \Vdash u=w: A .
$$

4. $I \Vdash A=B \Rightarrow I \Vdash B=A$

5. $I \Vdash A \& I \Vdash u=v: A \Rightarrow I \Vdash v=u: A$

Proof We prove the statement for " $\vdash$ " " instead of " $\vdash$ " by main induction on $\ell$ (i.e., we prove the statement for " $\vdash_{0}$ " before the statement for " $\vdash_{1}$ "); the statement for " $\vdash$ " follows then from Lemma 3.

Simultaneously by threefold induction on $I \Vdash_{\ell} A, I \Vdash_{\ell} B$, and $I \Vdash_{\ell} C$. (Alternatively by induction on the (natural) sum of the heights of $I \Vdash_{\ell} A, I \Vdash_{\ell} B$, and $I \Vdash_{\ell} C$; we only need to be able to apply the IH if the complexity of at least one derivation decreases and the others won't increase.) In the proof below we will omit $\ell$ to simplify notation, except in cases where the level matters.

(1) By distinguishing cases on $I \Vdash A=B$. We only give the argument for (1a) as (1b) is very similar except in case GL-E. The cases N-E and U-E are trivial.

Case PI-E Let $I \Vdash w:(x: A) \rightarrow B$ and we show $I \Vdash w:\left(x: A^{\prime}\right) \rightarrow B^{\prime}$. For $f: J \rightarrow I$ let $J \Vdash u: A^{\prime} f$; then by IH (since $J \Vdash A f=A^{\prime} f$ ) we get $J \Vdash u: A f$, and thus $J \Vdash w f u: B(f, x / u)$; again by IH we obtain $J \Vdash w f u: B^{\prime}(f, x / u)$. Now assume $J \Vdash u=v: A^{\prime} f$; so by IH, $J \Vdash u=v: A f$, and thus $J \Vdash w f u=w f v: B(f, x / u)$. Again by IH, we conclude $J \Vdash w f u=w f v: B^{\prime}(f, x / u)$. Thus we have proved $I \Vdash w:$ $\left(x: A^{\prime}\right) \rightarrow B^{\prime}$.

Case SI-E Let $I \Vdash w:(x: A) \times B$ and we show $I \Vdash w:\left(x: A^{\prime}\right) \times B^{\prime}$. We have $I \Vdash w .1: A$ and $I \Vdash w .2: B(x / w .1)$. So by $\mathrm{IH}, I \Vdash w .1: A^{\prime}$; moreover, we have $I \Vdash B(x / w .1)=B^{\prime}(x / w .1)$; so, again by $\mathrm{IH}$, we conclude with $I \Vdash w .2: B^{\prime}(x / w .1)$.

Case PA-E Let $I \Vdash u$ : Path $A a_{0} a_{1}$ and we show $I \Vdash u$ : Path $B b_{0} b_{1}$. Given $f: J \rightarrow I$ and $r \in \mathbb{F}(J)$ we have $J \Vdash u f r: A f$ and thus $J \Vdash u f r: B f$ by IH. We have to check that the endpoints match: $I \Vdash u 0=a_{0}: A$ by assumption; moreover, $I \Vdash a_{0}=b_{0}: A$, so by $\mathrm{IH}(3), I \Vdash u 0=b_{0}: A$, thus again using the $\mathrm{IH}, I \Vdash u 0=b_{0}: B$.

Case GL-E Abbreviate Glue $[\varphi \mapsto(T, w)] A$ by $D$, and Glue $\left[\varphi \mapsto\left(T^{\prime}, w^{\prime}\right)\right] A^{\prime}$ by $D^{\prime}$.

(1a) Let $I \Vdash u: D$, i.e., $I, \varphi \Vdash u: D$ and

$$
J \Vdash \text { unglue }\left[\varphi f \mapsto w^{\prime \prime}\right] u f=\text { unglue }[\varphi f \mapsto w f] u f: A f
$$

whenever $f: J \rightarrow I$ and $J, \varphi f \Vdash w^{\prime \prime}=w f$ : Equiv $T f A f$. Directly by IH we obtain $I, \varphi \Vdash u: D^{\prime}$. Now let $f: J \rightarrow I$ and $J, \varphi f \Vdash w^{\prime \prime}=w^{\prime} f:$ Equiv $T^{\prime} f A^{\prime} f$; by $\mathrm{IH}$, also $J, \varphi f \Vdash w^{\prime \prime}=w^{\prime} f$ : Equiv $T f A f$. Moreover, we have $J, \varphi f \Vdash w f=w^{\prime} f$ : Equiv $T f A f$, hence (1) gives (together with symmetry and transitivity, applicable by $\mathrm{IH}$ )

$$
\begin{aligned}
& J \Vdash \text { unglue }\left[\varphi f \mapsto w^{\prime \prime}\right] u f=\text { unglue }[\varphi f \mapsto w f] u f: A f, \quad \text { and } \\
& J \Vdash \text { unglue }\left[\varphi f \mapsto w^{\prime} f\right] u f=\text { unglue }[\varphi f \mapsto w f] u f: A f .
\end{aligned}
$$

Hence, transitivity and symmetry (which we can apply by $\mathrm{IH}$ ) give that the above left-hand sides are forced equal of type $A f$, applying the $\mathrm{IH}(1 \mathrm{~b})$ gives that they are forced equal of type $A^{\prime} f$, and thus $I \Vdash u: D^{\prime}$. 
(1b) Let $I \Vdash u=v: D$, so we have $I, \varphi \Vdash u=v: D$ and

$$
I \Vdash \text { unglue }[\varphi \mapsto w] u=\text { unglue }[\varphi \mapsto w] v: A
$$

By IH, we get $I, \varphi \Vdash u=v: D^{\prime}$ from $I, \varphi \Vdash u=v: D$. Note that we also have $I \Vdash u: D$ and $I \Vdash v: D$, and thus

$$
\begin{aligned}
& I \Vdash \text { unglue }[\varphi \mapsto w] u=\text { unglue }\left[\varphi \mapsto w^{\prime}\right] u: A, \quad \text { and } \\
& I \Vdash \text { unglue }[\varphi \mapsto w] v=\text { unglue }\left[\varphi \mapsto w^{\prime}\right] v: A
\end{aligned}
$$

and thus with (2) and transitivity and symmetry (which we can apply by $\mathrm{IH}$ ) we obtain $I \Vdash$ unglue $\left[\varphi \mapsto w^{\prime}\right] u=$ unglue $\left[\varphi \mapsto w^{\prime}\right] v: A$, hence also at type $A^{\prime}$ by IH. Therefore we proved $I \Vdash u=v: D^{\prime}$.

Case NI-E Let $I \Vdash u: A$; we have to show $I \Vdash u: B$.

Subcase $B$ is non-introduced. Then we have to show $J \Vdash u f: B f \downarrow$ for $f: J \rightarrow I$. We have $J \Vdash A f \downarrow=B f \downarrow$ and since $I \Vdash B$ is non-introduced, the derivation $J \Vdash B f \downarrow$ is shorter than $I \Vdash B$, and the derivation $J \Vdash A f \downarrow$ is not higher than $I \Vdash A$ by Lemma 6 . Moreover, also $J \Vdash u f$ : Af so by Lemma 6 (3) we get $J \Vdash u f: A f \downarrow$, and hence by IH, $J \Vdash u f: B f \downarrow$.

Subcase $B$ is introduced. We have $I \Vdash u: A \downarrow$ and $I \Vdash A \downarrow=B \downarrow$ but $B \downarrow$ is $B$, and $I \Vdash A \downarrow$ has a shorter derivation than $I \Vdash A$, so $I \Vdash u: B$ by $\mathrm{IH}$.

(2) Let us first handle the cases where $A, B$, or $C$ is non-introduced. It is enough to show $J \Vdash A f \downarrow=C f \downarrow$ (if $A$ and $C$ are both introduced, this entails $I \Vdash A=C$ for $f$ the identity). We have $J \Vdash A f \downarrow=B f \downarrow$ and $J \Vdash B f \downarrow=C f \downarrow$. None of the respective derivations get higher (by Lemma 6 ) but one gets shorter since one of the types is non-introduced. Thus the claim follows by IH.

It remains to look at the cases where all are introduced; in this case both equalities have to be derived by the same rule. We distinguish cases on the rule.

Case N-E Trivial. Case SI-E. Similar to PI-E below. Case PA-E and GL-E. Use the IH. Case U-E. Trivial.

Case PI-E Let use write $A$ as $\left(x: A^{\prime}\right) \rightarrow A^{\prime \prime}$ and similar for $B$ and $C$. We have $I \Vdash A^{\prime}=$ $B^{\prime}$ and $I \Vdash B^{\prime}=C^{\prime}$, and so by $\mathrm{IH}$, we get $I \Vdash A^{\prime}=C^{\prime}$; for $J \Vdash u: A^{\prime} f$ where $f: J \rightarrow I$ it remains to be shown that $J \Vdash A^{\prime \prime}(f, x / u)=C^{\prime \prime}(f, x / u)$. By IH, we also have $J \Vdash u: B^{\prime} f$, so we have

$$
J \Vdash A^{\prime \prime}(f, x / u)=B^{\prime \prime}(f, x / u) \quad \text { and } \quad J \Vdash B^{\prime \prime}(f, x / u)=C^{\prime \prime}(f, x / u)
$$

and can conclude by the IH.

(3) By cases on $I \Vdash A$. All cases follow immediately using the $\mathrm{IH}$, except for $\mathrm{N}-\mathrm{C}$ and $\mathrm{U}-\mathrm{C}$. In case $\mathrm{N}-\mathrm{C}$, we show transitivity by a side induction on the (natural) sum of the height of the derivations $I \Vdash u=v: \mathrm{N}$ and $I \Vdash v=w: \mathrm{N}$. If one of $u, v$, or $w$ is non-introduced, we get that one of the derivations $J \Vdash u f \downarrow=v f \downarrow: \mathrm{N}$ and $J \Vdash v f \downarrow=w f \downarrow: \mathrm{N}$ is shorter (and the other doesn't get higher), so by SIH, $J \Vdash u f \downarrow=w f \downarrow$ : N which entails $I \Vdash u=w: \mathrm{N}$. Otherwise, $I \Vdash u=v: \mathrm{N}$ and $I \Vdash v=w: \mathrm{N}$ have to be derived with the same rule and $I \Vdash u=w: \mathrm{N}$ easily follows (using the SIH in the successor case).

In case U-C, we have $I \Vdash_{1} u=v: \mathrm{U}$ and $I \Vdash_{1} v=w: \mathrm{U}$, i.e., $I \Vdash_{0} u=v$ and $I \Vdash_{0} v=w$. We want to show $I \Vdash_{1} u=w: \mathrm{U}$, i.e., $I \Vdash_{0} u=w$. But by $\operatorname{IH}(\ell)$, we can already assume the lemma is proven for $\ell=0$, hence can use transitivity and deduce $I \Vdash_{0} u=w$.

The proofs of (4) and (5) are by distinguishing cases and are straightforward. 
Remark 2 Now that we have established transitivity, proving computability for $\Pi$-types can also be achieved as follows. Given we have $I \Vdash(x: A) \rightarrow B$ and derivations $I \vdash w$ : $(x: A) \rightarrow B, I \vdash w^{\prime}:(x: A) \rightarrow B$, and $I \vdash w=w^{\prime}:(x: A) \rightarrow B$, then $I \Vdash w=w^{\prime}:(x: A) \rightarrow B$ whenever we have

$$
\forall f: J \rightarrow I \forall u, v\left(J \Vdash u=v: A f \Rightarrow J \Vdash w f u=w^{\prime} f v: B(f, x / u)\right) .
$$

(In particular, this gives $I \Vdash w:(x: A) \rightarrow B$ and $I \Vdash w^{\prime}:(x: A) \rightarrow B$.)

Likewise, given $I \vdash(x: A) \rightarrow B, I \vdash\left(x: A^{\prime}\right) \rightarrow B^{\prime}$, and $I \vdash(x: A) \rightarrow B=(x:$ $\left.A^{\prime}\right) \rightarrow B^{\prime}$, we get $I \Vdash(x: A) \rightarrow B=\left(x: A^{\prime}\right) \rightarrow B^{\prime}$ whenever $I \Vdash A=A^{\prime}$ and

$$
\forall f: J \rightarrow I \forall u, v\left(J \Vdash u=v: A f \Rightarrow J \Vdash B(f, x / u)=B^{\prime}(f, x / v)\right) .
$$

Lemma 8 1. $I \Vdash A \Rightarrow I \Vdash A=A \downarrow$

2. $I \Vdash u: \mathrm{N} \Rightarrow I \Vdash u=u \downarrow: \mathrm{N}$

Proof (1) We already proved $I \Vdash A \downarrow$ in Lemma 6 (1). By induction on $I \Vdash A$. All cases where $A$ is an introduction are immediate since then $A \downarrow$ is $A$. It only remains the case NI-C:

$A$ n.i.

$\frac{\forall f: J \rightarrow I(A f ! \& J \Vdash A f \downarrow) \quad \forall f: J \rightarrow I \forall g: K \rightarrow J(K \Vdash A f \downarrow g=A f g \downarrow)}{I \Vdash A}$ NI-C

We now show $I \Vdash A=A \downarrow$; since $A$ is non-introduced we have to show $J \Vdash A f \downarrow=(A \downarrow f) \downarrow$ for $f: J \rightarrow I$. $I \Vdash A \downarrow$ has a shorter derivation than $I \Vdash A$, thus so has $J \Vdash A \downarrow f$; hence by $\mathrm{IH}, J \Vdash A \downarrow f=(A \downarrow f) \downarrow$. We also have $J \Vdash A \downarrow f=A f \downarrow$ by definition of $I \Vdash A$, and thus we obtain $J \Vdash A f \downarrow=(A \downarrow f) \downarrow$ using symmetry and transitivity.

(2) Similar, by induction on $I \Vdash u: N$.

Lemma 9 (Expansion Lemma) Let $I \Vdash_{\ell} A$ and $I \vdash u: A$; then:

$$
\frac{\forall f: J \rightarrow I\left(u f !^{A f} \& J \Vdash_{\ell} u f \downarrow^{A f}: A f\right) \quad \forall f: J \rightarrow I\left(J \Vdash_{\ell} u f \downarrow=u \downarrow f: A f\right)}{I \Vdash_{\ell} u: A \& I \Vdash_{\ell} u=u \downarrow: A}
$$

In particular, if $I \vdash u \succ_{\mathrm{s}} v: A$ and $I \Vdash_{\ell} v: A$, then $I \Vdash_{\ell} u: A$ and $I \Vdash_{\ell} u=v: A$.

Proof By induction on $I \Vdash A$. We will omit the level annotation $\ell$ whenever it is inessential.

Case N-C. We have to show $K \Vdash u f \downarrow g=u f g \downarrow: \mathrm{N}$ for $f: J \rightarrow I$ and $g: K \rightarrow J$; we have $J \Vdash u f \downarrow=u \downarrow f: \mathrm{N}$, thus $K \Vdash u f \downarrow g=u \downarrow f g: \mathrm{N}$. Moreover, $K \Vdash u \downarrow f g=u f g \downarrow$ : $\mathrm{N}$ by assumption, and thus by transitivity $K \Vdash u f \downarrow g=u \downarrow f g=u f g \downarrow$ : N. (Likewise one shows that the data in the premise of the lemma is closed under substitution.)

$I \Vdash u=u \downarrow: \mathrm{N}$ holds by Lemma 6 (2).

Case PI-C. First, let $J \Vdash a: A f$ for $f: J \rightarrow I$. We have

$$
K \vdash(u f a) g \succ(u f g) \downarrow(a g): B(f g, x / a g)
$$

for $g: K \rightarrow J$, and also $K \Vdash(u f g) \downarrow(a g): B(f g, x / a g)$ and we have the compatibility condition

$$
\begin{aligned}
K \Vdash(u f a) g \downarrow & =((u f g) \downarrow)(a g)=(u f \downarrow g)(a g) \\
& =(u f \downarrow a) g=(u f a) \downarrow g: B(f g, x / a g),
\end{aligned}
$$

so by IH, $J \Vdash u f a: B(f, x / a)$ and $J \Vdash u f a=u f \downarrow a: B(f, x / a)$. Since also $J \Vdash u f \downarrow=$ $u \downarrow f:((x: A) \rightarrow B) f$ we also get $J \Vdash u f a=u \downarrow f a: B(f, x / a)$. 
Now if $J \Vdash a=b: A f$, we also have $J \Vdash a: A f$ and $J \Vdash b: A f$, so like above we get $J \Vdash u f a=u f \downarrow a: B(f, x / a)$ and $J \Vdash u f b=u f \downarrow b: B(f, x / b)$ (and thus also $J \Vdash u f b=u f \downarrow b: B(f, x / a))$. Moreover, $J \Vdash u f \downarrow a=u f \downarrow b: B(f, x / a)$ and hence we can conclude $J \Vdash$ uf $a=u f b: B(f, x / a)$ by transitivity and symmetry. Thus we showed both $I \Vdash u:(x: A) \rightarrow B$ and $I \Vdash u=u \downarrow:(x: A) \rightarrow B$.

Case SI-C. Clearly we have $(u .1 f) \downarrow=(u f \downarrow) .1, J \Vdash(u f \downarrow) .1: A f$, and

$$
J \Vdash(u .1 f) \downarrow=(u f \downarrow) .1=(u \downarrow f) .1=(u \downarrow .1) f=(u .1) \downarrow f: A f
$$

so the IH gives $I \Vdash u .1: A$ and $I \Vdash u .1=(u \downarrow) .1: A$. Likewise $(u .2 f) \downarrow=(u f \downarrow) .2$ and $J \Vdash(u f \downarrow) .2: B(f, x / u f \downarrow .1)$, hence also $J \Vdash(u f \downarrow) .2: B(f, x / u f .1)$; as above one shows $J \Vdash(u .2 f) \downarrow=u .2 \downarrow f: B(f, x / u f .1)$, applying the IH once more to obtain $I \Vdash u .2=u \downarrow .2: B(x / u .1))$ which was what remained to be proven.

Case PA-C. Let us write Path $A v w$ for the type and let $f: J \rightarrow I, r \in \mathbb{I}(J)$, and $g: K \rightarrow J$. We have

$$
K \vdash(u f r) g \succ(u f g) \downarrow(r g): A f g
$$

and $K \Vdash(u f g) \downarrow(r g): A f g$; moreover,

$$
K \Vdash(u f r) g \downarrow=(u f g) \downarrow(r g)=(u f \downarrow g)(r g)=(u f \downarrow r) g=(u f r) \downarrow g: A f g .
$$

Thus by IH, $J \Vdash u f r: A f$ and

$$
J \Vdash u f r=u f \downarrow r=u \downarrow f r: A f .
$$

So we obtain $I \Vdash u 0=u \downarrow 0=v: A$ and $I \Vdash u 1=u \downarrow 1=w: A$, and hence $I \Vdash u$ : Path $A v w ; I \Vdash u=u \downarrow$ : Path $A v w$ follows from (3).

Case GL-C. Abbreviate Glue $[\varphi \mapsto(T, w)] A$ by $B$. Note that we have $\varphi \neq 1$. First, we claim that for any $f: J \rightarrow I, J \Vdash b: B f$, and $J, \varphi f \Vdash w^{\prime}=w f:$ Equiv $T f$ Af,

$$
J, \varphi f \Vdash \text { unglue }\left[\varphi f \mapsto w^{\prime}\right] b=w^{\prime} .1 b: A f .
$$

(In particular both sides are computable.) Indeed, for $g: K \rightarrow J$ with $\varphi f g=1$ we have that

$$
\left.K \vdash \text { (unglue }\left[\varphi f \mapsto w^{\prime}\right] b\right) g \succ_{\mathrm{s}} w^{\prime} g .1(b g): A f g
$$

and $K \Vdash w^{\prime} g .1(b g):$ Afg since $I, \varphi \Vdash B=T$ (which follows from Lemma 8 (1)). Thus by $\mathrm{IH}(J \Vdash A f$ has a shorter derivation than $I \Vdash B), K \Vdash$ (unglue $\left.\left[\varphi f \mapsto w^{\prime}\right] b\right) g=$ $\left(w^{\prime} .1 b\right) g: A f g$ as claimed.

Next, let $f: J \rightarrow I$ such that $\varphi f=1$; then using the IH $(J \Vdash B f$ has a shorter derivation than $I \Vdash B$ ), we get $J \Vdash u f: B f$ and $J \Vdash u f=u f \downarrow: B f$, and hence also $J \Vdash u f=u \downarrow f: B f$ (since $J \Vdash u f \downarrow=u \downarrow f: B f$ ). That is, we proved

$$
I, \varphi \Vdash u: B \quad \text { and } \quad I, \varphi \Vdash u=u \downarrow: B .
$$

We will now first show

$$
J \Vdash \text { unglue }\left[\varphi f \mapsto w^{\prime}\right] u f=\left(\text { unglue }\left[\varphi f \mapsto w^{\prime}\right] u f\right) \downarrow: A f
$$

for and $f: J \rightarrow I$ and $J, \varphi f \Vdash w^{\prime}=w f$ : Equiv $T f A f$. We can assume that w.l.o.g. $\varphi f \neq 1$, since if $\varphi f=1, J \Vdash u f: B f$ by (5), and (6) follows from (4) noting that its right-hand side is the reduct. We will use the IH to show (6), so let us analyze the reduct:

$$
\text { (unglue } \left.\left[\varphi f \mapsto w^{\prime}\right] u f\right) g \downarrow= \begin{cases}\left(w^{\prime} g .1\right)(u f g) & \text { if } \varphi f g=1, \\ \text { unglue }\left[\varphi f \mapsto w^{\prime} g\right](u f g \downarrow) & \text { otherwise. }\end{cases}
$$


where $g: K \rightarrow J$. In either case, the reduct is computable: in the first case, use (5) and $J \Vdash w^{\prime} .1: T \rightarrow A$ together with the observation $I, \varphi \Vdash B=T$; in the second case this follows from $J \Vdash u f g \downarrow: B f g$. In order to apply the IH, it remains to verify

$$
\left.K \Vdash \text { (unglue }\left[\varphi f \mapsto w^{\prime}\right] u f\right) g \downarrow=\left(\text { unglue }\left[\varphi f \mapsto w^{\prime}\right] u f\right) \downarrow g: A f g .
$$

In case $\varphi f g \neq 1$, we have

$$
\begin{array}{rlrl}
K \Vdash & \text { unglue }\left[\varphi f g \mapsto w^{\prime} g\right](u f g \downarrow) & \\
& =\text { unglue }[\varphi f g \mapsto w f g](u f g \downarrow) & & \text { since } K \Vdash u f g \downarrow: B f g \\
& =\text { unglue }[\varphi f g \mapsto w f g](u f \downarrow g) & & \text { since } K \Vdash u f g \downarrow=u f \downarrow g: B f g \\
& =\text { unglue }\left[\varphi f g \mapsto w^{\prime} g\right](u f \downarrow g): A f g & & \text { since } K \Vdash u f \downarrow g: B f g
\end{array}
$$

which is what we had to show in this case. In case $\varphi f g=1$, we have to prove

$$
K \Vdash\left(w^{\prime} g .1\right)(u f g)=\text { unglue }\left[\varphi f \mapsto w^{\prime} g\right](u f \downarrow g): A f g .
$$

But by (5) we have $K \Vdash u f g=u f g \downarrow=u f \downarrow g: B f g$, so also

$$
K \Vdash\left(w^{\prime} g .1\right)(u f g)=\left(w^{\prime} g .1\right)(u f \downarrow g): A f g,
$$

so (8) follows from (4) using $J \Vdash u f \downarrow: B f$. This concludes the proof of (6).

As $w^{\prime}$ could have been $w f$ we also get

$$
J \Vdash \text { unglue }[\varphi f \mapsto w f] u f=(\text { unglue }[\varphi f \mapsto w f] u f) \downarrow: A f \text {. }
$$

In order to prove $I \Vdash u: B$ it remains to check that the left-hand side of (6) is forced equal to the left-hand side of (9); so we can simply check this for the respective right-hand sides: in case $\varphi f=1$, these are $w^{\prime} .1 u f$ and $w f .1 u f$, respectively, and hence forced equal since $J \Vdash w^{\prime}=w f$ : Equiv $T f A f$; in case $\varphi f \neq 1$, we have to show

$$
J \Vdash \text { unglue }\left[\varphi f \mapsto w^{\prime}\right](u f \downarrow)=\text { unglue }[\varphi f \mapsto w f](u f \downarrow): A f
$$

which simply follows since $J \Vdash u f \downarrow: B f$.

In order to prove $I \Vdash u=u \downarrow: B$ it remains to check

$$
I \Vdash \text { unglue }[\varphi \mapsto w] u=\text { unglue }[\varphi \mapsto w](u \downarrow): A,
$$

but this is (9) in the special case where $f$ is the identity.

Case U-C. Let us write $B$ for $u$. We have to prove $I \Vdash_{1} B: \cup$ and $I \Vdash_{1} B=B \downarrow: U$, i.e., $I \Vdash_{0} B$ and $I \Vdash_{0} B=B \downarrow$. By Lemma 8 (1), it suffices to prove the former. For $f: J \rightarrow I$ we have

$$
J \vdash B f \succ B f \downarrow^{\cup}: \cup
$$

and hence also

$$
J \vdash B f \succ B f \downarrow^{\cup}
$$

i.e., $B f$ !, and $B f \downarrow$ is $B f \downarrow \downarrow^{U}$; since $J \Vdash_{1} B f \downarrow: U$ we have $J \Vdash_{0} B f \downarrow$, and likewise $J \Vdash_{0} B f \downarrow=B \downarrow f$. Moreover, if also $g: K \rightarrow J$, we obtain $K \Vdash_{0} B f g \downarrow=B \downarrow f g$ from the assumption. Hence $K \vdash_{0} B f \downarrow g=B \downarrow f g=B f g \downarrow$, therefore $I \Vdash_{0} B$ what we had to show.

Case NI-C. Then $I \Vdash A \downarrow$ has a shorter derivation than $I \Vdash A$; moreover, for $f: J \rightarrow I$ we have $J \vdash u f \succ u f \downarrow^{A f}: A f$ so also $J \vdash u f \succ u f \downarrow^{A f}: A \downarrow f$ since $J \vdash A f=A \downarrow f$. By Lemma 6 (1), $I \Vdash A=A \downarrow$ so also $J \Vdash u f \downarrow: A \downarrow f$ and $J \Vdash u f \downarrow=u \downarrow f: A \downarrow f$, and 
hence by $\mathrm{IH}, I \Vdash u: A \downarrow$ and $I \Vdash u=u \downarrow: A \downarrow$, so also $I \Vdash u: A$ and $I \Vdash u=u \downarrow: A$ using $I \Vdash A=A \downarrow$ again.

\section{Soundness}

The aim of this section is to prove canonicity as stated in the introduction. We will do so by showing that each computable instance of a judgment derived in cubical type theory is computable (allowing free name variables) - this is the content of the Soundness Theorem below.

We first extend the computability predicates to contexts and substitutions.

$\Vdash \Gamma$ assuming $\Gamma \vdash$.

$$
\frac{\Vdash \Gamma \quad i \notin \operatorname{dom}(\Gamma)}{\Vdash \diamond} \quad \frac{\Vdash \Gamma, i: \mathbb{I}}{\Vdash \Gamma, \varphi: \mathbb{F}}
$$

$\Vdash \Gamma$

$\frac{\forall I \forall \sigma(I \Vdash \sigma: \Gamma \Rightarrow I \Vdash A \sigma) \quad \forall I \forall \sigma, \tau(I \Vdash \sigma=\tau: \Gamma \Rightarrow I \Vdash A \sigma=A \tau) \quad x \notin \operatorname{dom}(\Gamma)}{\Vdash \Gamma, x: A}$

$I \Vdash \sigma: \Gamma$ by induction on $\Vdash \Gamma$ assuming $I \vdash \sigma: \Gamma$.

$$
\begin{array}{cc}
\frac{I \Vdash \sigma: \Gamma \quad r \in \mathbb{I}(I)}{I \Vdash(): \diamond} & \frac{I \Vdash \sigma: \Gamma \quad \varphi \sigma=1}{I \Vdash(\sigma, i / r): \Gamma, i: \mathbb{I}} \quad \frac{I \Vdash \sigma: \Gamma, \varphi}{\frac{I \Vdash \sigma: \Gamma \quad I \Vdash u: A \sigma}{I \Vdash(\sigma, x / u): \Gamma, x: A}}
\end{array}
$$

$I \Vdash \sigma=\tau: \Gamma$ by induction on $\Vdash \Gamma$, assuming $I \Vdash \sigma: \Gamma, I \Vdash \tau: \Gamma$, and $I \vdash \sigma=\tau: \Gamma$.

$$
\begin{gathered}
\frac{I \Vdash \sigma=\tau: \Gamma \quad r \in \mathbb{I}(I)}{I \Vdash()=(): \diamond} \quad \frac{I \Vdash \sigma=\tau: \Gamma \quad \varphi \sigma=\varphi \tau=1}{I \Vdash(\sigma, i / r)=(\tau, i / r): \Gamma, i: \mathbb{I}} \quad \frac{I \Vdash \sigma=\tau: \Gamma, \varphi}{I \Vdash \sigma=\tau: \Gamma \quad I \Vdash u=v: A \sigma} \\
\frac{I \Vdash(\sigma, x / u)=(\tau, x / v): \Gamma, x: A}{I \Vdash(x)}
\end{gathered}
$$

We write $I \Vdash r: \mathbb{I}$ for $r \in \mathbb{I}(I), I \Vdash r=s: \mathbb{I}$ for $r=s \in \mathbb{I}(I)$, and likewise $I \Vdash \varphi: \mathbb{F}$ for $\varphi \in \mathbb{F}(I), I \Vdash \varphi=\psi: \mathbb{F}$ for $\varphi=\psi \in \mathbb{F}(I)$. In the next definition we allow $A$ to be $\mathbb{F}$ or $\mathbb{I}$, and also correspondingly for $a$ and $b$ to range over interval and face lattice elements. 


\section{Definition 1}

$$
\begin{array}{lll}
\Gamma \models & : \Leftrightarrow & \Vdash \Gamma \\
\Gamma \models A=B & : \Leftrightarrow & \Gamma \vdash A=B \& \Gamma \models \& \\
& & \forall I, \sigma, \tau(I \Vdash \sigma=\tau: \Gamma \Rightarrow I \Vdash A \sigma=B \tau) \\
\Gamma \models A & : \Leftrightarrow & \Gamma \vdash A \& \Gamma \models A=A \\
\Gamma \models a=b: A & : \Leftrightarrow & \Gamma \vdash a=b: A \& \Gamma \models A \& \\
& & \forall I, \sigma, \tau(I \Vdash \sigma=\tau: \Gamma \Rightarrow I \Vdash a \sigma=b \tau: A \sigma) \\
\Gamma \models a: A & : \Leftrightarrow & \Gamma \vdash a: A \& \Gamma \models a=a: A \\
\Gamma \models \sigma=\tau: \Delta & : \Leftrightarrow & \Gamma \vdash \sigma=\tau: \Delta \& \Gamma \models \& \Delta \models \& \\
& & \forall I, \delta, \gamma(I \Vdash \delta=\gamma: \Gamma \Rightarrow I \Vdash \sigma \delta=\tau \gamma: \Delta) \\
\Gamma \models \sigma: \Delta & : \Leftrightarrow & \Gamma \vdash \sigma: \Delta \& \Gamma \models \sigma=\sigma: \Delta
\end{array}
$$

Remark 3 1. For each $I$ we have $\Vdash I$, and $J \Vdash \sigma: I$ iff $\sigma: J \rightarrow I$; likewise, $J \Vdash \sigma=$ $\tau: I$ iff $\sigma=\tau$.

2. For computability of contexts and substitutions monotonicity and partial equivalence properties hold analogous to computability of types and terms.

3. Given $\Vdash \Gamma$ and $I \Vdash \sigma=\tau: \Gamma$, then for any $\Gamma \vdash \varphi: \mathbb{F}$ we get $\varphi \sigma=\varphi \tau \in \mathbb{F}(I)$ since $\varphi \sigma$ and $\varphi \tau$ only depend on the name assignments of $\sigma$ and $\tau$ which have to agree by $I \Vdash \sigma=\tau: \Gamma$. Similarly for $\Gamma \vdash r: \mathbb{I}$.

4. The definition of " $\models$ " slightly deviates from the approach we had in the definition of " $\vdash$ " as, say, $\Gamma \models A$ is defined in terms of $\Gamma \models A=A$. Note that by the properties we already established about " $\vdash$ " we get that $\Gamma \models A=B$ implies $\Gamma \models A$ and $\Gamma \models B$ (given we know $\Gamma \vdash A$ and $\Gamma \vdash B$, respectively); and, likewise, $\Gamma \models a=b: A$ entails $\Gamma \models a: A$ and $\Gamma \models b: A$ (given $\Gamma \vdash a: A$ and $\Gamma \vdash b: A$, respectively). Also, note that in the definition of, say, $\Gamma \models A$, the condition

$$
\forall I, \sigma, \tau(I \Vdash \sigma=\tau: \Gamma \Rightarrow I \Vdash A \sigma=A \tau)
$$

implies

$$
\forall I, \sigma(I \Vdash \sigma: \Gamma \Rightarrow I \Vdash A \sigma) .
$$

In fact, we will often have to establish the latter condition first when showing the former.

5. $I \models A=B$ iff $I \Vdash A=B$, and $I \models a=b: A$ iff $I \Vdash A$ and $I \Vdash a=b: A$; moreover, given $I \models A$ and $I, x: A \vdash B$, then $I, x: A \models B$ iff

$$
\begin{aligned}
& \forall f: J \rightarrow I \forall u(J \Vdash u: A f \Rightarrow J \Vdash B(f, x / u)) \quad \& \\
& \forall f: J \rightarrow I \forall u, v(J \Vdash u=v: A f \Rightarrow J \Vdash B(f, x / u)=B(f, x / v))
\end{aligned}
$$

(Note that the second formula in the above display implies the first.) Thus the premises of PI-C and SI-C are simply $I \models A$ and $I, x: A \models B$. Also, $I, \varphi \Vdash A=B$ iff $I, \varphi \models A=B$; and $I, \varphi \models a=b: A$ iff $I, \varphi \Vdash A$ and $I, \varphi \Vdash a=b: A$.

6. By Lemma 7 we get that $\Gamma \models \cdot=\cdot, \Gamma \models \cdot=\cdot: A$, and $\Gamma \models \cdot=\cdot: \Delta$ are partial equivalence relations.

Theorem 1 (Soundness) $\Gamma \vdash \mathscr{J} \Rightarrow \Gamma \models \mathscr{J}$

The proof of the Soundness Theorem spans the rest of this section. We will mainly state and prove congruence rules as the proof of the other rules are special cases. 
Lemma 10 The context formation rules are sound:

$$
\overline{\diamond \models} \quad \frac{\Gamma \models i \notin \operatorname{dom}(\Gamma)}{\Gamma, i: \mathbb{I} \models} \quad \frac{\Gamma \models \varphi: \mathbb{F}}{\Gamma, \varphi \models} \quad \frac{\Gamma \models A \quad x \notin \operatorname{dom}(\Gamma)}{\Gamma, x: A \models}
$$

Proof Immediately by definition.

Lemma 11 Given $\Gamma \models, \Gamma \vdash r: \mathbb{I}, \Gamma \vdash s: \mathbb{I}, \Gamma \vdash \varphi: \mathbb{F}$, and $\Gamma \vdash \psi: \mathbb{F}$ we have:

1. $\Gamma \vdash r=s: \mathbb{I} \Rightarrow \Gamma \models r=s: \mathbb{I}$

2. $\Gamma \vdash \varphi=\psi: \mathbb{F} \Rightarrow \Gamma \models \varphi=\psi: \mathbb{F}$

Proof (1) By virtue of Remark 3 (3) it is enough to show $r \sigma=s \sigma \in \mathbb{I}(I)$ for $I \Vdash \sigma: \Gamma$. But then by applying the substitution $I \vdash \sigma: \Gamma$ we get $I \vdash r \sigma=s \sigma: \mathbb{I}$, and thus $r \sigma=s \sigma \in \mathbb{I}(I)$ since the context $I$ does not contain restrictions. The proof of (2) is analogous.

Lemma 12 The rule for type conversion is sound:

$$
\frac{\Gamma \models a=b: A \quad \Gamma \models A=B}{\Gamma \models a=b: B}
$$

Proof Suppose $I \Vdash \sigma=\tau: \Gamma$. By assumption we have $I \Vdash a \sigma=b \tau: A \sigma$. Moreover also $I \Vdash \sigma=\sigma: \Gamma$, so $I \Vdash A \sigma=B \sigma$, and hence $I \Vdash a \sigma=b \tau: B \sigma$ by Lemma 7 which was what we had to prove.

\section{Lemma 13}

$$
\begin{gathered}
\frac{\Gamma \models \sigma=\tau: \Delta \quad \Delta \models A=B}{\Gamma \models A \sigma=B \tau} \quad \frac{\Gamma \models \sigma=\tau: \Delta \quad \Delta \models a=b: A}{\Gamma \models a \sigma=b \tau: A \sigma} \\
\frac{\Gamma \models \sigma=\tau: \Delta \quad \Delta \models \delta=\gamma: \Xi}{\Gamma \models \delta \sigma=\gamma \tau: \Xi}
\end{gathered}
$$

Proof Immediate by definition.

Lemma 14 The rules for $\Pi$-types are sound:

1. $\frac{\Gamma \models A=A^{\prime} \quad \Gamma, x: A \models B=B^{\prime}}{\Gamma \models(x: A) \rightarrow B=\left(x: A^{\prime}\right) \rightarrow B^{\prime}}$

2. $\frac{\Gamma \models A=A^{\prime} \quad \Gamma, x: A \models t=t^{\prime}: B}{\Gamma \models \lambda x: A . t=\lambda x: A^{\prime} \cdot t^{\prime}:(x: A) \rightarrow B}$

3. $\frac{\Gamma \models w=w^{\prime}:(x: A) \rightarrow B \quad \Gamma \models u=u^{\prime}: A}{\Gamma \models w u=w^{\prime} u^{\prime}: B(x / u)}$

4. $\frac{\Gamma, x: A \models t: B \quad \Gamma \models u: A}{\Gamma \models(\lambda x: A . t) u=t(x / u): B(x / u)}$

5. $\frac{\Gamma \models w:(x: A) \rightarrow B \quad \Gamma \models w^{\prime}:(x: A) \rightarrow B \quad \Gamma, x: A \models w x=w^{\prime} x: B}{\Gamma \models w=w^{\prime}:(x: A) \rightarrow B}$ 
Proof Abbreviate $(x: A) \rightarrow B$ by $C$. We will make use of Remark 2 .

(1) It is enough to prove this in the case where $\Gamma$ is of the form $I$, in which case this directly follows by PI-E.

(2) Suppose $\Gamma \models A=A^{\prime}$ and $\Gamma, x: A \models t=t^{\prime}: B$; this entails $\Gamma, x: A \models B$. For $I \Vdash \sigma=\tau: \Gamma$ we show $I \Vdash(\lambda x: A . t) \sigma=\left(\lambda x: A^{\prime} . t^{\prime}\right) \tau: C \sigma$. For this let $J \Vdash u=v: A \sigma f$ where $f: J \rightarrow I$. Then also $J \Vdash u=v: A^{\prime} \tau f$,

$$
\begin{aligned}
& J \vdash(\lambda x: A . t) \sigma f u \succ_{\mathrm{s}} t(\sigma f, x / u): B(\sigma f, x / u), \quad \text { and } \\
& J \vdash\left(\lambda x: A^{\prime} . t^{\prime}\right) \tau f v \succ_{\mathrm{s}} t^{\prime}(\tau f, x / v): B(\tau f, x / v) .
\end{aligned}
$$

Moreover, $J \Vdash(\sigma f, x / u)=(\tau f, x / v): \Gamma, x: A$, and so $J \Vdash B(\sigma f, x / u)=B(\tau f, x / v)$ and

$$
J \Vdash t(\sigma f, x / u)=t^{\prime}(\tau f, x / v): B(\sigma f, x / u)
$$

which gives

$$
\begin{aligned}
& J \Vdash(\lambda x: A . t) \sigma f u=t(\sigma f, x / u): B(\sigma f, x / u), \quad \text { and } \\
& J \Vdash\left(\lambda x: A^{\prime} . t^{\prime}\right) \tau f v=t^{\prime}(\tau f, x / v): B(\tau f, x / v),
\end{aligned}
$$

by applying the Expansion Lemma twice, and thus also

$$
J \Vdash(\lambda x: A . t) \sigma f u=\left(\lambda x: A^{\prime} . t^{\prime}\right) \tau f v: B(\sigma f, x / u)
$$

what we had to show.

(3) For $I \Vdash \sigma=\tau: \Gamma$ we get $I \Vdash w \sigma=w^{\prime} \tau: C \sigma$ and $I \Vdash u \sigma=u^{\prime} \tau: A \sigma$; so also $I \Vdash w \sigma: C \sigma$, therefore $I \Vdash(w u) \sigma=w \sigma u^{\prime} \tau=\left(w^{\prime} u^{\prime}\right) \tau: B(\sigma, x / u)$.

(4) Given $I \Vdash \sigma=\tau: \Delta$ we get, like in (2), $I \Vdash(\lambda x: A . t) \sigma u \sigma=t(\sigma, x / u \sigma)$ : $B(\sigma, x / u \sigma)$ using the Expansion Lemma; moreover, $I \Vdash(\sigma, x / u \sigma)=(\tau, x / u \tau): \Gamma, x: A$, hence

$$
I \Vdash(\lambda x: A . t) \sigma u \sigma=t(\sigma, x / u \sigma)=t(\tau, x / u \tau): B(\sigma, x / u \sigma) .
$$

(5) Suppose $I \Vdash \sigma=\tau: \Gamma$ and $J \Vdash u: A \sigma f$ for $f: J \rightarrow I$. We have to show $J \Vdash w \sigma f u=w^{\prime} \tau f u: B(\sigma f, x / u)$. We have

$$
J \Vdash(\sigma f, x / u)=(\tau f, x / u): \Gamma, x: A
$$

and thus, by the assumption $\Gamma, x: A \models w x=w^{\prime} x: B$, we get

$$
J \Vdash(w x)(\sigma f, x / u)=\left(w^{\prime} x\right)(\tau f, x / u): B(\sigma f, x / u) .
$$

Since $x$ does neither appear in $w$ nor in $w^{\prime}$ this was what we had to prove.

Lemma 15 The rules for $\Sigma$-types are sound:

1. $\frac{\Gamma \models A=A^{\prime} \quad \Gamma, x: A \models B=B^{\prime}}{\Gamma \models(x: A) \times B=\left(x: A^{\prime}\right) \times B^{\prime}}$
2. $\frac{\Gamma, x: A \models B \quad \Gamma \models u=u^{\prime}: A \quad \Gamma \models v=v^{\prime}: B(x / u)}{\Gamma \models(u, v)=\left(u^{\prime}, v^{\prime}\right):(x: A) \times B}$

3. $\frac{\Gamma, x: A \models B \quad \Gamma \models w=w^{\prime}:(x: A) \times B}{\Gamma \models w .1=w^{\prime} .1: A}$

$\Gamma \models w .2=w^{\prime} .2: B(x / w .1)$ 
4. $\frac{\Gamma, x: A \models B \quad \Gamma \models u: A \quad \Gamma \models v: B(x / u)}{\Gamma \models(u, v) .1=u: A}$

$$
\Gamma \models(u, v) .2=v: B(x / u)
$$

$$
\Gamma, x: A \models B \quad \Gamma \models w:(x: A) \times B
$$

5. $\frac{\Gamma \models w^{\prime}:(x: A) \times B \quad \Gamma \models w .1=w^{\prime} .1: A \quad \Gamma \models w .2=w^{\prime} .2: B(x / w .1)}{\Gamma \models w=w^{\prime}:(x: A) \times B}$

Lemma 16 Given $I, x: \mathrm{N} \models C$ we have:

1. $\frac{I \Vdash u: \mathrm{N} \quad I \Vdash z: C(x / 0) \quad I \Vdash s:(x: \mathrm{N}) \rightarrow C \rightarrow C(x / \mathrm{S} x)}{I \Vdash \text { natrec } u z s: C(x / u)}$

$I \Vdash u=u^{\prime}: \mathrm{N}$
2. $\frac{I \Vdash z=z^{\prime}: C(x / 0) \quad I \Vdash s=s^{\prime}:(x: \mathrm{N}) \rightarrow C \rightarrow C(x / \mathrm{S} x)}{I \Vdash \operatorname{natrec} u z s=\text { natrec } u^{\prime} z^{\prime} s^{\prime}: C(x / u)}$

Proof By simultaneous induction on $I \Vdash u: \mathrm{N}$ and $I \Vdash u=u^{\prime}: \mathrm{N}$.

Case $I \Vdash 0: \mathrm{N}$. We have $I \vdash \operatorname{natrec} 0 z s \succ_{\mathrm{s}} z: C(x / 0)$ so (1) follows from the Expansion Lemma.

Case $I \Vdash 0=0:$ N. (2) immediately follows from (1) and $I \Vdash z=z^{\prime}: C(x / 0)$.

Case $I \Vdash \mathrm{S} u: \mathrm{N}$ from $I \Vdash u: \mathrm{N}$. We have

$$
I \vdash \operatorname{natrec}(\mathrm{S} u) z s \succ_{\mathrm{s}} s u(\text { natrec } u z s): C(x / \mathrm{S} u)
$$

and $I \Vdash s u$ (natrec $u z s): C(x / \mathrm{S} u)$ by $\mathrm{IH}$, and using that $u$ and $s$ are computable. Hence we are done by the Expansion Lemma.

Case $I \Vdash \mathrm{S} u=\mathrm{S} u^{\prime}: \mathrm{N}$ from $I \Vdash u=u^{\prime}: \mathrm{N}$. (2) follows from (1) and $I \Vdash s=s^{\prime}:(x$ : $\mathrm{N}) \rightarrow C \rightarrow C(x / \mathrm{S} x), I \Vdash u=u^{\prime}: \mathrm{N}$, and the $\mathrm{IH}$.

Case $I \Vdash u: \mathrm{N}$ for $u$ non-introduced. For $f: J \rightarrow I$ we have

$$
J \vdash(\text { natrec } u z s) f \succ \operatorname{natrec}(u f \downarrow) z f s f: C(f, x / u f \downarrow) .
$$

Moreover, we have $I \Vdash u f \downarrow$ and $I \Vdash u f \downarrow=u \downarrow f: \mathrm{N}$ with a shorter derivation (and thus also $J \Vdash C(f, x / u f \downarrow)=C(x / u \downarrow) f)$, hence by $\mathrm{IH}$

$$
\begin{aligned}
& J \Vdash \text { natrec }(u f \downarrow) z f s f: C(x / u \downarrow) f, \quad \text { and } \\
& J \Vdash \text { natrec }(u f \downarrow) z f s f=(\operatorname{natrec}(u \downarrow) z s) f: C(x / u \downarrow) f,
\end{aligned}
$$

which yields the claim by the Expansion Lemma.

Case $I \Vdash u=u^{\prime}: \mathrm{N}$ for $u$ or $u^{\prime}$ non-introduced. We have

$$
I \Vdash \operatorname{natrec} u z s=\operatorname{natrec}(u \downarrow) z s: C(x / u)
$$

by either (1) (if $u$ is non-introduced) or by reflexivity (if $u$ is an introduction); likewise for $u^{\prime}$. So with the IH for $I \Vdash u \downarrow=u^{\prime} \downarrow: \mathrm{N}$ we obtain

$$
I \Vdash \operatorname{natrec} u z s=\operatorname{natrec}(u \downarrow) z s=\operatorname{natrec}\left(u^{\prime} \downarrow\right) z^{\prime} s^{\prime}=\operatorname{natrec} u^{\prime} z^{\prime} s^{\prime}: C(x / u)
$$

what we had to show.

We write $\underline{n}$ for the numeral $\mathrm{S}^{n} 0$ where $n \in \mathbb{N}$. 
Lemma 17 If $I \Vdash u: \mathrm{N}$, then $I \Vdash u=\underline{n}: \mathrm{N}$ (and hence also $I \vdash u=\underline{n}: \mathrm{N}$ ) for some $n \in \mathbb{N}$.

Proof By induction on $I \Vdash u: \mathrm{N}$. The cases for zero and successor are immediate. In case $u$ is non-introduced, then $I \Vdash u \downarrow=\underline{n}$ for some $n \in \mathbb{N}$ by IH. By Lemma 8 (2) and transitivity we conclude $I \Vdash u=\underline{n}: \mathrm{N}$.

Lemma $18 I \Vdash \cdot=\cdot: \mathrm{N}$ is discrete, i.e., if $I \Vdash u: \mathrm{N}, I \Vdash v: \mathrm{N}$, and $J \Vdash u f=v g: \mathrm{N}$ for some $f, g: J \rightarrow I$, then $I \Vdash u=v: \mathrm{N}$.

Proof By Lemma 17, we have $I \Vdash u=\underline{n}: \mathrm{N}$ and $I \Vdash v=\underline{m}: \mathrm{N}$ for some $n, m \in \mathbb{N}$, and thus $J \Vdash \underline{n}=u f=v g=\underline{m}: \mathrm{N}$, i.e., $J \Vdash \underline{n}=\underline{m}: \mathrm{N}$ and hence $n=m$ which yields $I \Vdash u=v: \mathrm{N}$.

Lemma 19 The rules for Path-types are sound:

1. $\frac{\Gamma \models A=A^{\prime} \quad \Gamma \models u=u^{\prime}: A \quad \Gamma \models v=v^{\prime}: A}{\Gamma \models \text { Path } A u v=\text { Path } A^{\prime} u^{\prime} v^{\prime}}$

2. $\frac{\Gamma \models A \quad \Gamma, i: \mathbb{I} \models t=t^{\prime}: A}{\Gamma \models\langle i\rangle t=\langle i\rangle t^{\prime}: \text { Path } A t(i 0) t(i 1)}$

3. $\frac{\Gamma \models w=w^{\prime}: \text { Path } A u v \quad \Gamma \models r=r^{\prime}: \mathbb{I}}{\Gamma \models w r=w^{\prime} r^{\prime}: A}$

4. $\frac{\Gamma \models w: \text { Path } A u v}{\Gamma \models w 0=u: A \quad \Gamma \models w 1=v: A}$

5. $\frac{\Gamma \models A \quad \Gamma, i: \mathbb{I} \models t: A \quad \Gamma \models r: \mathbb{I}}{\Gamma \models(\langle i\rangle t) r=t(i / r): A}$

6. $\frac{\Gamma \models w: \text { Path } A u v \quad \Gamma \models w^{\prime}: \text { Path } A u v \quad \Gamma, i: \mathbb{I} \models w i=w^{\prime} i: A}{\Gamma \models w=w^{\prime}: \text { Path } A u v}$

Proof (1) Follows easily by definition.

(2) For $I \Vdash \sigma=\sigma^{\prime}: \Gamma$ we have to show

$$
I \Vdash(\langle i\rangle t) \sigma=\left(\langle i\rangle t^{\prime}\right) \sigma^{\prime} \text { : Path } A \sigma t(\sigma, i / 0) t(\sigma, i / 1) .
$$

For $f: J \rightarrow I$ and $r \in \mathbb{I}(J)$ we have $J \Vdash(\sigma f, i / r)=\left(\sigma^{\prime} f, i / r\right): \Gamma, i: \mathbb{I}$ and

$$
\begin{aligned}
& J \vdash(\langle i\rangle t)(\sigma f) r \succ_{\mathrm{s}} t(\sigma f, i / r): A \sigma f, \quad \text { and } \\
& J \vdash\left(\langle i\rangle t^{\prime}\right)\left(\sigma^{\prime} f\right) r \succ_{\mathrm{s}} t^{\prime}\left(\sigma^{\prime} f, i / r\right): A \sigma^{\prime} f,
\end{aligned}
$$

and moreover $J \Vdash t(\sigma f, i / r)=t^{\prime}\left(\sigma^{\prime} f, i / r\right): A \sigma f$ and $J \Vdash A \sigma f=A \sigma^{\prime} f$ by assumption. Hence the Expansion Lemma yields

$$
\begin{aligned}
& J \Vdash(\langle i\rangle t)(\sigma f) r=t(\sigma f, i / r): A \sigma f, \quad \text { and } \\
& J \Vdash\left(\langle i\rangle t^{\prime}\right)\left(\sigma^{\prime} f\right) r=t^{\prime}\left(\sigma^{\prime} f, i / r\right): A \sigma f,
\end{aligned}
$$

in particular also, say $J \Vdash(\langle i\rangle t) \sigma 0=t(\sigma, i / 0): A \sigma$ and $J \Vdash\left(\langle i\rangle t^{\prime}\right) \sigma^{\prime} 0=t^{\prime}\left(\sigma^{\prime}, i / 0\right)=$ $t(\sigma, i / 0): A \sigma$. And hence (10) follows. 
(3) Supposing $I \Vdash \sigma=\sigma^{\prime}: \Gamma$ we have to show $I \Vdash(w \sigma)(r \sigma)=\left(w^{\prime} \sigma^{\prime}\right)\left(r^{\prime} \sigma\right): A \sigma$. We have $I \Vdash w \sigma=w^{\prime} \sigma^{\prime}$ : Path $A \sigma u \sigma v \sigma$ and $r \sigma=r^{\prime} \sigma^{\prime}$, hence the claim follows by definition.

(4) Let $I \Vdash \sigma=\sigma^{\prime}: \Gamma$; we have to show, say, $I \Vdash w \sigma 0=u \sigma^{\prime}: A \sigma$. First, we get $I \Vdash w \sigma$ : Path $A \sigma u \sigma v \sigma$. Since $\Gamma \models w$ : Path $A u v$ we also have $\Gamma \models$ Path $A u v$, hence

$$
I \Vdash \text { Path } A \sigma u \sigma v \sigma=\text { Path } A \sigma^{\prime} u \sigma^{\prime} v \sigma^{\prime} .
$$

Hence we also obtain $I \Vdash w \sigma$ : Path $A \sigma^{\prime} u \sigma^{\prime} v \sigma^{\prime}$, and thus $I \Vdash w \sigma 0=u \sigma^{\prime}: A \sigma^{\prime}$. But (11) also yields $I \Vdash A \sigma=A \sigma^{\prime}$ by definition, so $I \Vdash w \sigma 0=u \sigma^{\prime}: A \sigma$ what we had to show.

(5) Similar to (2) using the Expansion Lemma.

(6) For $I \Vdash \sigma=\sigma^{\prime}: \Gamma, f: J \rightarrow I$, and $r \in \mathbb{I}(J)$, we have $J \Vdash(\sigma f, i / r)=\left(\sigma^{\prime} f, i / r\right)$ : $\Gamma, i: \mathbb{I}$, and thus

$$
J \Vdash(w i)(\sigma f, i / r)=\left(w^{\prime} i\right)\left(\sigma^{\prime} f, i / r\right): A \sigma f .
$$

But $(w i)(\sigma f, i / r)$ is $w \sigma f r$, and $\left(w^{\prime} i\right)\left(\sigma^{\prime} f, i / r\right)$ is $w^{\prime} \sigma^{\prime} f r$, so (12) is what we had to show.

Lemma 20 Let $\varphi_{i} \in \mathbb{F}(I)$ and $\varphi_{1} \vee \cdots \vee \varphi_{n}=1$.

1. Let $I, \varphi_{i} \Vdash_{\ell} A_{i}$ and $I, \varphi_{i} \wedge \varphi_{j} \Vdash_{\ell} A_{i}=A_{j}$ for all $i, j$; then

(a) $I \Vdash_{\ell}\left[\varphi_{1} A_{1}, \ldots, \varphi_{n} A_{n}\right]$, and

(b) $I \Vdash_{\ell}\left[\varphi_{1} A_{1}, \ldots, \varphi_{n} A_{n}\right]=A_{k}$ whenever $\varphi_{k}=1$.

2. Let $I \Vdash_{\ell} A, I, \varphi_{i} \Vdash_{\ell} t_{i}: A$, and $I, \varphi_{i} \wedge \varphi_{j} \Vdash_{\ell} t_{i}=t_{j}:$ A for all $i$, $j$; then

(a) $I \Vdash_{\ell}\left[\varphi_{1} t_{1}, \ldots, \varphi_{n} t_{n}\right]: A$, and

(b) $I \Vdash_{\ell}\left[\varphi_{1} t_{1}, \ldots, \varphi_{n} t_{n}\right]=t_{k}:$ A whenever $\varphi_{k}=1$.

Proof (1) Let us abbreviate $\left[\varphi_{1} A_{1}, \ldots, \varphi_{n} A_{n}\right]$ by $A$. Since $A$ is non-introduced, we have to show $J \Vdash A f \downarrow$ and $J \Vdash A f \downarrow=A \downarrow f$. For the former observe that $A f \downarrow$ is $A_{k} f$ with $k$ minimal such that $\varphi_{k} f=1$. For the latter use that $J \Vdash A_{k} f=A_{l} f$ if $\varphi_{k} f=1$ and $\varphi_{l}=1$, since $I, \varphi_{k} \wedge \varphi_{l} \Vdash A_{k}=A_{l}$.

(2) Let us write $t$ for $\left[\varphi_{1} t_{1}, \ldots, \varphi_{n} t_{n}\right]$. By virtue of the Expansion Lemma, it suffices to show $J \Vdash t f \downarrow: A f$ and $K \Vdash t f \downarrow=t \downarrow f: A f$. The proof is just like the proof for types given above.

Lemma 21 Given $\Gamma \models \varphi_{1} \vee \cdots \vee \varphi_{n}=1: \mathbb{F}$, then:

$$
\frac{\Gamma, \varphi_{1} \models \mathscr{J} \ldots \Gamma, \varphi_{n} \models \mathscr{J}}{\Gamma \models \mathscr{J}}
$$

Proof Let $\varphi=\varphi_{1} \vee \cdots \vee \varphi_{n}$. Say if $\mathscr{J}$ is a typing judgment of the form $A$. For $I \Vdash \sigma: \Gamma$ we have $\varphi \sigma=1$, so $\varphi_{k} \sigma=1$ for some $k$, hence $I \Vdash A \sigma$ by $\Gamma, \varphi_{k} \models A$. Now let $I \Vdash \sigma=\tau: \Gamma$; then $\varphi_{i} \sigma=\varphi_{i} \tau$ ( $\sigma$ and $\tau$ assign the same elements to the interval variables), so $\varphi \sigma=\varphi \tau=1$ yields $\varphi_{k} \sigma=\varphi_{k} \tau=1$ for some common $k$ and thus $I \Vdash A \sigma=A \tau$ follows from $\Gamma, \varphi_{k} \models A$. The other judgment forms are similar.

For $I \Vdash A$ and $I, \varphi \Vdash v: A$ we write $I \Vdash u: A[\varphi \mapsto v]$ for $I \Vdash u: A$ and $I, \varphi \Vdash u=v: A$. And likewise $I \Vdash u=w: A[\varphi \mapsto v]$ means $I \Vdash u=w: A$ and $I, \varphi \Vdash u=v: A$ (in this case also $I, \varphi \Vdash w=v: A$ follows). We use similar notations for for " $=$ ". 
Lemma 22 Given $\varphi \in \mathbb{F}(I)$ and $I \Vdash_{\ell} A, I, \varphi \Vdash_{\ell} T$, and $I, \varphi \Vdash_{\ell} w:$ Equiv $T$ A, and write $B$ for Glue $[\varphi \mapsto(T, w)]$ A. Then:

1. $I \Vdash_{\ell} B$ and $I, \varphi \Vdash_{\ell} B=T$.

2. If $I \Vdash_{\ell} A=A^{\prime}, I, \varphi \Vdash_{\ell} T=T^{\prime}, I, \varphi \Vdash_{\ell} w=w^{\prime}$ : Equiv $T A$, then $I \Vdash_{\ell} B=$ Glue $\left[\varphi \mapsto\left(T^{\prime}, w^{\prime}\right)\right] A^{\prime}$.

3. If $I \Vdash_{\ell} u: B$ and $I, \varphi \Vdash_{\ell} w=w^{\prime}:$ Equiv $T A$, then $I \Vdash_{\ell}$ unglue $\left[\varphi \mapsto w^{\prime}\right] u: A[\varphi \mapsto$ $\left.w^{\prime} .1 u\right]$ and $I \Vdash_{\ell}$ unglue $[\varphi \mapsto w] u=$ unglue $\left[\varphi \mapsto w^{\prime}\right] u: A$.

4. If $I \Vdash_{\ell} u=u^{\prime}: B$, then

$$
I \Vdash_{\ell} \text { unglue }[\varphi \mapsto w] u=\text { unglue }[\varphi \mapsto w] u^{\prime}: A .
$$

5. If $I, \varphi \Vdash_{\ell} t=t^{\prime}: T$ and $I \Vdash_{\ell} a=a^{\prime}: A[\varphi \mapsto w .1 t]$, then

(a) $I \Vdash_{\ell}$ glue $[\varphi \mapsto t] a=$ glue $\left[\varphi \mapsto t^{\prime}\right] a^{\prime}: B$,

(b) $I, \varphi \Vdash_{\ell}$ glue $[\varphi \mapsto t] a=t: T$, and

(c) $I \Vdash_{\ell}$ unglue $[\varphi \mapsto w]($ glue $[\varphi \mapsto t] a)=a: A$.

6. If $I \Vdash_{\ell} u: B$, then $I \Vdash_{\ell} u=$ glue $[\varphi \mapsto u]$ (unglue $\left.[\varphi \mapsto w] u\right): B$.

Proof (1) Let us first prove $I, \varphi \Vdash B$ and $I, \varphi \Vdash B=T$; but in $I, \varphi, \varphi$ becomes 1 so w.l.o.g. let us assume $\varphi=1$; then $B$ is non-introduced and $I \vdash B \succ_{\mathrm{s}} T$ so $I \Vdash B$ from $I \Vdash T$. For $I \Vdash B=T$ we have to show $J \Vdash B f \downarrow=T f \downarrow$ for $f: J \rightarrow I$. But $B f \downarrow$ is $T f$ so this is an instance of Lemma 8.

It remains to prove $I \Vdash B$ in case where $\varphi \neq 1$; for this use GL-C with the already proven $I, \varphi \Vdash B$.

(2) In case $\varphi \neq 1$ we only have to show $I, \varphi \Vdash B=B^{\prime}$ and can apply GL-E. But restricted to $I, \varphi, \varphi$ becomes 1 and hence we only have to prove the statement for $\varphi=1$. But then by (1) we have $I \Vdash B=T=T^{\prime}=B^{\prime}$.

(3) In case $\varphi \neq 1, I \Vdash$ unglue $\left[\varphi \mapsto w^{\prime}\right] u: A$ and

$$
I \Vdash_{\ell} \text { unglue }[\varphi \mapsto w] u=\text { unglue }\left[\varphi \mapsto w^{\prime}\right] u: A
$$

are immediate by definition. Using the Expansion Lemma (and $I \vdash$ unglue $\left[\varphi \mapsto w^{\prime}\right] u \succ_{\mathrm{s}}$ $w^{\prime} .1 u: A$ for $\left.\varphi=1\right)$ we obtain $I, \varphi \Vdash$ unglue $\left[\varphi \mapsto w^{\prime}\right] u=w^{\prime} .1 u: A$, which also shows $I \Vdash$ unglue $\left[\varphi \mapsto w^{\prime}\right] u: A$ as well as (13) in case $\varphi=1$.

(4) In case $\varphi \neq 1$, this is by definition. For $\varphi=1$ we have

$$
I \Vdash \text { unglue }[\varphi \mapsto w] u=w .1 u=w .1 u^{\prime}=\text { unglue }[\varphi \mapsto w] u^{\prime}: A .
$$

(5) Let us write $b$ for glue $[\varphi \mapsto t] a$, and $b^{\prime}$ for glue $\left[\varphi \mapsto t^{\prime}\right] a^{\prime}$. We first show $I \Vdash b: B$ and $I, \varphi \Vdash b=t: B$ (similarly for $b^{\prime}$ ).

In case $\varphi=1, I \vdash b \succ_{\mathrm{s}} t: T$ so by the Expansion Lemma $I \Vdash b: T$ and $I \Vdash b=t: T$, and hence also $I \Vdash b: B$ and $I \Vdash b=t: B$ by (1). This also proves (5b).

Let now $\varphi$ be arbitrary; we claim

$$
I \Vdash \text { unglue }[\varphi \mapsto w] b: A \text { and } I \Vdash \text { unglue }[\varphi \mapsto w] b=a: A
$$

[and thus proving (5c)]. We will apply the Expansion Lemma to do so; for $f: J \rightarrow I$ let us analyze the reduct of (unglue $[\varphi \mapsto w] b) f$ :

$$
\text { (unglue }[\varphi \mapsto w] b) f \downarrow= \begin{cases}w f .1 b f & \text { if } \varphi f=1, \\ a f & \text { otherwise. }\end{cases}
$$


Note that, if $\varphi f=1$, we have as in the case for $\varphi=1, J \Vdash b f=t f: B f$ and hence $J \Vdash w f .1 b f=w f .1 t f=a f: A f$. This ensures $J \Vdash$ (unglue $[\varphi \mapsto w] b) f \downarrow=$ (unglue $[\varphi \mapsto w] b) \downarrow f: A$, and thus the Expansion Lemma applies and we obtain $I \Vdash$ unglue $[\varphi \mapsto w] b=$ (unglue $[\varphi \mapsto w] b) \downarrow: A$; but as we have seen in either case, $\varphi=1$ or not, $I \Vdash$ (unglue $[\varphi \mapsto w] b) \downarrow=a: A$ proving the claim.

Let now be $\varphi \neq 1, f: J \rightarrow I$, and $J \Vdash w^{\prime}=w f$ : Equiv $T f A f$. We can use the claim for $B f$ and Glue $\left[\varphi f \mapsto\left(T f, w^{\prime}\right)\right] A f$ (which is forced equal to $B f$ by (2)) and obtain both

$$
J \Vdash \text { unglue }[\varphi f \mapsto w f] b f=a f: A f \quad \text { and } \quad J \Vdash \text { unglue }\left[\varphi f \mapsto w^{\prime}\right] b f=a f: A f,
$$

so the left-hand sides are equal; moreover, $I, \varphi \Vdash b: B$ (as in the case $\varphi=1$ ), and hence $I \Vdash b: B$. Likewise one shows $I \Vdash b^{\prime}: B$.

It remains to show $I \Vdash b=b^{\prime}: B$. If $\varphi=1$, we already showed $I \Vdash b=t: T$ and $I \Vdash b^{\prime}=t^{\prime}: T$, so the claim follows from $I \Vdash t=t^{\prime}: T$ and $I \Vdash T=B$. Let us now assume $\varphi \neq 1$. We immediately get $I, \varphi \Vdash b=t=t^{\prime}=b^{\prime}: B$ as for $\varphi=1$. Moreover, we showed above that $I \Vdash$ unglue $[\varphi \mapsto w] b=a: A$ and $I \Vdash$ unglue $[\varphi \mapsto w] b^{\prime}=a^{\prime}: A$. Hence we obtain

$$
I \Vdash \text { unglue }[\varphi \mapsto w] b=\text { unglue }[\varphi \mapsto w] b^{\prime}: A
$$

from $I \Vdash a=a^{\prime}: A$.

(6) In case $\varphi=1$, this follows from (5b). In case $\varphi \neq 1$, we have to show

$$
\begin{gathered}
I \Vdash \text { unglue }[\varphi \mapsto w] u=\text { unglue }[\varphi \mapsto w] \text { (glue }[\varphi \mapsto u](\text { unglue }[\varphi \mapsto w] u)): A \quad \text { and } \\
I, \varphi \Vdash u=\text { glue }[\varphi \mapsto u](\text { unglue }[\varphi \mapsto w] u): T \text {. }
\end{gathered}
$$

The former is an instance of (5c); the latter follows from $(5 b)$.

Lemma 23 Let $B$ be Glue $[\varphi \mapsto(T, w)] A$ and suppose $I \Vdash B$ is derived via GL-C, then also $I, \varphi \Vdash T$ and the derivations of $I, \varphi \Vdash T$ are all proper sub-derivations of $I \Vdash B$ (and hence shorter).

Proof We have the proper sub-derivations $I, \varphi \Vdash B$. For each $f: J \rightarrow I$ with $\varphi f=1$, we have that $B f$ is non-introduced with reduct $T f$ so the derivation of $J \Vdash B f$ has a derivation of $J \Vdash T f$ as sub-derivation according to NI-C.

For the next proof we need a small syntactic observation. Given $\Gamma \vdash \alpha: \mathbb{F}$ irreducible, there is an associated substitution $\bar{\alpha}: \Gamma_{\alpha} \rightarrow \Gamma$ where $\Gamma_{\alpha}$ skips the names of $\alpha$ and applies a corresponding $\bar{\alpha}$ to the types and restrictions (e.g., if $\Gamma$ is $i: \mathbb{I}, x: A, j: \mathbb{I}, \varphi$ and $\alpha$ is $(i=0)$, then $\Gamma_{\alpha}$ is $\left.x: A(i 0), j: \mathbb{I}, \varphi(i 0)\right)$. Since $\alpha \bar{\alpha}=1$ we even have $\bar{\alpha}: \Gamma_{\alpha} \rightarrow \Gamma, \alpha$. The latter has an inverse (w.r.t. judgmental equality) given by the projection $\mathrm{p}: \Gamma, \alpha \rightarrow \Gamma_{\alpha}$ (i.e., $\mathrm{p}$ assigns each variable in $\Gamma_{\alpha}$ to itself): in the context $\Gamma, \alpha, \bar{\alpha} \mathrm{p}$ is the identity, and $\mathrm{p} \bar{\alpha}$ is the identity since the variables in $\Gamma_{\alpha}$ are not changed by $\bar{\alpha}$.

Remark 4 We can use the above observation to show that the condition $I, \varphi \vdash \mathscr{J}$ in the definition of $I, \varphi \Vdash_{\ell} \mathscr{J}$ (in Sect. 3) already follows from the other, i.e., $J \Vdash_{\ell} \mathscr{J} f$ for all $f: J \rightarrow I, \varphi$ : We have to show $I, \alpha \vdash \mathscr{J}$ for each irreducible $\alpha \leq \varphi$. But we have $I_{\alpha} \Vdash_{\ell} \mathscr{J} \bar{\alpha}$ by the assumption and $\bar{\alpha}: I_{\alpha} \rightarrow I, \varphi$, and hence $I_{\alpha} \vdash \mathscr{J} \bar{\alpha}$. Substituting along p: $I, \alpha \rightarrow I_{\alpha}$ yields $I, \alpha \vdash \mathscr{J}$.

Theorem 2 Compositions are computable, i.e., for $\varphi \in \mathbb{F}(I)$ and $i \notin \operatorname{dom}(I)$ : 
1. $\frac{I, i \Vdash A \quad I, i, \varphi \Vdash u: A \quad I \Vdash u_{0}: A(i 0)[\varphi \mapsto u(i 0)]}{I \Vdash \operatorname{comp}^{i} A[\varphi \mapsto u] u_{0}: A(i 1)[\varphi \mapsto u(i 1)]}$

$I \Vdash \operatorname{comp}^{i} A[\varphi \mapsto u] u_{0}=\left(\operatorname{comp}^{i} A[\varphi \mapsto u] u_{0}\right) \downarrow: A(i 1)$

2. $\frac{I, i \Vdash A \quad I, i, \varphi \Vdash u=v: A \quad I \Vdash u_{0}=v_{0}: A(i 0)[\varphi \mapsto u(i 0)]}{I \Vdash \operatorname{comp}^{i} A[\varphi \mapsto u] u_{0}=\operatorname{comp}^{i} A[\varphi \mapsto v] v_{0}: A(i 1)}$

3. $\frac{I, i \Vdash A=B \quad I, i, \varphi \Vdash u: A \quad I \Vdash u_{0}: A(i 0)[\varphi \mapsto u(i 0)]}{I \Vdash \operatorname{comp}^{i} A[\varphi \mapsto u] u_{0}=\operatorname{comp}^{i} B[\varphi \mapsto u] u_{0}: A(i 1)}$

Proof By simultaneous induction on $I, i \Vdash A$ and $I, i \Vdash A=B$. Let us abbreviate $\operatorname{comp}^{i} A[\varphi \mapsto u] u_{0}$ by $u_{1}$, and $\operatorname{comp}^{i} A[\varphi \mapsto v] v_{0}$ by $v_{1}$. The second conclusion of (1) holds since in each case we will use the Expansion Lemma and in particular also prove $I \Vdash u_{1} \downarrow: A(i 1)$.

Let us first make some preliminary remarks. Given the induction hypothesis holds for $I, i \Vdash A$ we also know that filling operations are admissible for $I, i \Vdash A$, i.e.:

$$
\frac{I, i \Vdash A \quad I, i, \varphi \Vdash u: A \quad I \Vdash u_{0}: A(i 0)[\varphi \mapsto u(i 0)]}{I, i \Vdash \text { fill }^{i} A[\varphi \mapsto u] u_{0}: A\left[\varphi \mapsto u,(i=1) \mapsto u_{1}\right]}
$$

To see this, recall the explicit definition of filling

$$
\text { fill }^{i} A[\varphi \mapsto u] u_{0}=\operatorname{comp}^{j} A(i / i \wedge j)\left[\varphi \mapsto u(i / i \wedge j),(i=0) \mapsto u_{0}\right] u_{0}
$$

where $j$ is fresh. The derivation of $I, i, j \Vdash A(i / i \wedge j)$ isn't higher than the derivation of $I, i \Vdash A$ so we have to check, with $u^{\prime}=\left[\varphi u(i / i \wedge j),(i=0) u_{0}\right]$ and $A^{\prime}=A(i / i \wedge j)$,

$$
I, i, j, \varphi \vee(i=0) \Vdash u^{\prime}: A^{\prime} \quad \text { and } \quad I, i, \varphi \vee(i=0) \Vdash u^{\prime}(j 0)=u_{0}: A(i 0) .
$$

To check the former, we have to show

$$
I, i, j, \varphi \wedge(i=0) \Vdash u(i / i \wedge j)=u_{0}: A^{\prime}
$$

in order to apply Lemma 20. So let $f: J \rightarrow I, i, j$ with $\varphi f=1$ and $f(i)=0$; then as $\varphi$ doesn't contain $i$ and $j$, also $\varphi(f-i, j)=1$ for $f-i, j: J \rightarrow I$ being the restriction of $f$, so by assumption $J \Vdash u(i 0)(f-i, j)=u_{0}(f-i, j): A(i 0)(f-i, j)$. Clearly, $(i 0)(f-i, j)=(i / i \wedge j) f$ so the claim follows.

Let us now check the right-hand side equation of (15): by virtue of Lemma 21 we have to check the equation in the contexts $I, i, \varphi$ and $I, i,(i=0)$; but $I, i, \varphi \Vdash u^{\prime}(j 0)=u(i 0)=$ $u_{0}: A(i 0)$ and $I, i,(i=0) \Vdash u^{\prime}(j 0)=u_{0}: A(i 0)$ by Lemma 20.

And likewise the filling operation preserves equality.

Case N-C. First, we prove that

$$
I, \varphi, i: \mathbb{I} \vdash u=u_{0}: \mathrm{N} .
$$

To show this, it is enough to prove $I, \alpha, i: \mathbb{I} \vdash u=u_{0}: \mathrm{N}$ for each $\alpha \leq \varphi$ irreducible. Let $\bar{\alpha}: I_{\alpha} \rightarrow I$ be the associated face substitution. We have $I_{\alpha}, i \Vdash u(\bar{\alpha}, i / i): \mathrm{N}$ and also $I_{\alpha} \Vdash u(\bar{\alpha}, i / 0)=u_{0} \bar{\alpha}: \mathrm{N}$ since $\varphi \bar{\alpha}=1$. By discreteness of N (Lemma 18),

$$
I_{\alpha}, i \Vdash u(\bar{\alpha}, i / i)=u_{0} \bar{\alpha}: \mathrm{N},
$$

therefore $I_{\alpha}, i \vdash u(\bar{\alpha}, i / i)=u_{0} \bar{\alpha}: \mathrm{N}$, i.e., $I_{\alpha}, i \vdash u \bar{\alpha}=u_{0} \bar{\alpha}: \mathrm{N}$ with $\bar{\alpha}$ considered as substitution $I_{\alpha}, i \rightarrow I, i$ and $u_{0}$ weakened to $I, i$. Hence $I, \alpha, i: \mathbb{I} \vdash u=u_{0}: \mathrm{N}$ by the observation preceding the statement of the theorem. 
Second, we prove that

$$
I, \varphi \Vdash u(i 1)=u_{0}: \mathrm{N} .
$$

$I, \varphi \vdash u(i 1)=u_{0}: \mathrm{N}$ immediately follows from (16). For $f: J \rightarrow I$ with $\varphi f=1$ we have to show $J \Vdash u(i 1) f=u_{0} f: \mathrm{N}$; since $\varphi f=1$ we get $J \Vdash u(i 0) f=u_{0} f: \mathrm{N}$ by assumption, i.e., $J \Vdash u(f, i / j)(j 0)=u_{0}(f, i / j)(j 0): \mathrm{N}$ (where $u_{0}$ is weakened to $I, j$ and $j$ fresh). By discreteness of $\mathrm{N}$, we obtain $J, j \Vdash u(f, i / j)=u_{0}(f, i / j): \mathrm{N}$ and hence $J \Vdash u(f, i / 1)=u_{0}(f, i / 1): \mathrm{N}$, i.e., $J \Vdash u(i 1) f=u_{0} f: \mathrm{N}$.

We now prove the statements simultaneously by a side induction on $I \Vdash u_{0}: \mathrm{N}$ and $I \Vdash u_{0}=v_{0}: \mathrm{N}$.

Subcase $I \Vdash 0: \mathrm{N}$. By (16) it follows that $I \vdash u_{1} \succ_{\mathrm{s}} 0: \mathrm{N}$, and hence $I \Vdash u_{1}: \mathrm{N}$ and $I \Vdash u_{1}=0: \mathrm{N}$ by the Expansion Lemma. Thus also $I, \varphi \Vdash u_{1}=u(i 1): \mathrm{N}$ by (17).

Subcase $I \Vdash \mathrm{S} u_{0}^{\prime}: \mathrm{N}$ from $I \Vdash u_{0}^{\prime}: \mathrm{N}$ with $u_{0}=\mathrm{S} u_{0}^{\prime}$. By (16) it follows that

$$
I \vdash u_{1} \succ_{\mathrm{S}} \mathrm{S}\left(\operatorname{comp}^{i} \mathrm{~N}[\varphi \mapsto \operatorname{pred} u] u_{0}^{\prime}\right): \mathrm{N} .
$$

From $I, \varphi \Vdash \mathrm{S} u_{0}^{\prime}=u(i 0): \mathrm{N}$ we get $I, \varphi \Vdash u_{0}^{\prime}=\operatorname{pred}\left(\mathrm{S} u_{0}^{\prime}\right)=\operatorname{pred} u(i 0): \mathrm{N}$ by Lemma 16 and thus by $\mathrm{SIH}, I \Vdash \operatorname{comp}^{i} \mathrm{~N}[\varphi \mapsto \operatorname{pred} u] u_{0}^{\prime}: \mathrm{N}[\varphi \mapsto(\operatorname{pred} u)(i 1)]$; hence $I \Vdash u_{1}: \mathrm{N}$ and $I \Vdash u_{1}=\mathrm{S}\left(\operatorname{comp}^{i} \mathrm{~N}[\varphi \mapsto \operatorname{pred} u] u_{0}^{\prime}\right): \mathrm{N}$ by the Expansion Lemma. Thus also

$$
I, \varphi \Vdash u_{1}=\mathrm{S}(\operatorname{pred} u(i 1))=\mathrm{S}\left(\operatorname{pred}\left(\mathrm{S} u_{0}^{\prime}\right)\right)=\mathrm{S} u_{0}^{\prime}=u(i 1): \mathrm{N}
$$

using (17).

Subcase $u_{0}$ is non-introduced. We use the Expansion Lemma: for each $f: J \rightarrow I$

$$
u_{1} f \downarrow=\operatorname{comp}^{j} \mathrm{~N}[\varphi f \mapsto u(f, i / j)]\left(u_{0} f \downarrow\right)
$$

the right-hand side is computable by $\mathrm{SIH}$, and this results in a compatible family of reducts by SIH, since we have $K \Vdash u_{0} f \downarrow g=u_{0} f g \downarrow: \mathrm{N}$. Thus we get $I \Vdash u_{1}: \mathrm{N}$ and $I \Vdash u_{1}=$ $u_{1} \downarrow: \mathrm{N}$. By SIH, $I, \varphi \Vdash u_{1} \downarrow=u(i 1): \mathrm{N}$ and thus also $I, \varphi \Vdash u_{1}=u(i 1): \mathrm{N}$.

Subcase $I \Vdash 0=0: \mathrm{N}$. Like above we get that $I \Vdash u_{1}=0=v_{1}: \mathrm{N}$.

Subcase $I \Vdash S u_{0}^{\prime}=S v_{0}^{\prime}: \mathrm{N}$ from $I \Vdash u_{0}^{\prime}=v_{0}^{\prime}: \mathrm{N}$. Follows from the SIH $I \Vdash$ $\operatorname{comp}^{i} \mathrm{~N}[\varphi \mapsto \operatorname{pred} u] u_{0}^{\prime}=\operatorname{comp}^{i} \mathrm{~N}[\varphi \mapsto \operatorname{pred} v] v_{0}^{\prime}: \mathrm{N}$ like above.

Subcase $I \Vdash u_{0}=v_{0}: \mathrm{N}$ and $u_{0}$ or $v_{0}$ is non-introduced. We have to show $J \Vdash u_{1} f \downarrow=$ $v_{1} f \downarrow: \mathrm{N}$ for $f: J \rightarrow I$. We have $J \Vdash u_{0} f \downarrow=v_{0} f \downarrow: \mathrm{N}$ with a shorter derivation, thus by $\mathrm{SIH}$

$$
J \Vdash \operatorname{comp}^{j} \mathrm{~N}[\varphi f \mapsto u(f, i / j)]\left(u_{0} f \downarrow\right)=\operatorname{comp}^{j} \mathrm{~N}[\varphi f \mapsto v(f, i / j)]\left(v_{0} f \downarrow\right): \mathrm{N}
$$

which is what we had to show.

Case PI-C. Let us write $(x: A) \rightarrow B$ for the type under consideration. (1) In view of the Expansion Lemma, the reduction rule for composition at $\Pi$-types (which is closed under substitution), and Lemma 14 (2) and (5), it suffices to show

$$
\begin{gathered}
I, x: A(i 1) \models \operatorname{comp}^{i} B(x / \bar{x})[\varphi \mapsto u \bar{x}]\left(u_{0} \bar{x}(i 0)\right): B(i 1), \text { and } \\
I, x: A(i 1), \varphi \models \operatorname{comp}^{i} B(x / \bar{x})[\varphi \mapsto u \bar{x}]\left(u_{0} \bar{x}(i 0)\right)=u(i 1) x: B(i 1),
\end{gathered}
$$

where $x^{\prime}=$ fill $^{i} A(i / 1-i)[] x$ and $\bar{x}=x^{\prime}(i / 1-i)$. By IH, we get $I, x: A(i 1), i: \mathbb{I} \models \bar{x}: A$ and $I, x: A(i 1) \models \bar{x}(i 1)=x: A(i 1)$, i.e.,

$$
\begin{array}{r}
I, x: A(i 1), i: \mathbb{I} \models \text { fill }^{i} A(i / 1-i)[] x: A(i / 1-i), \text { and } \\
I, x: A(i 1) \models\left(\text { fill }^{i} A(i / 1-i)[] x\right)(i 0)=x: A(i 1) .
\end{array}
$$


To see (20), let $J \Vdash(f, x / a)=(f, x / b): I, x: A(i 1)$, i.e., $f: J \rightarrow I$ and $J \Vdash a=b$ : $A(i 1) f$; for $j$ fresh, we have $J, j \Vdash A(f, i / 1-j)$ (note that $(i 1) f=(f, i / 1-j)(j 0))$ and we get

$$
J, j \Vdash \text { fill }^{j} A(f, i / 1-j)[] a=\text { fill }^{j} A(f, i / 1-j)[] b: A(f, i / 1-j)
$$

by $\mathrm{IH}$, i.e., $J, j \Vdash x^{\prime}(f, x / a, i / j)=x^{\prime}(f, x / a, i / j): A(f, i / 1-j)$, and hence for $r \in \mathbb{I}(J)$

$$
J \Vdash x^{\prime}(f, x / a, i / r)=x^{\prime}(f, x / b, i / r):(A(i / 1-i))(f, x / a, i / r) .
$$

Thus we get $I, x: A(i 1) \models u_{0} \bar{x}(i 0): B(i 0)(x / \bar{x}(i 0)), I, x: A(i 1), \varphi, i: \mathbb{I} \models u \bar{x}:$ $B(x / \bar{x})$, and

$$
I, x:: A(i 1), \varphi \models u_{0} \bar{x}(i 0)=u(i 0) \bar{x}(i 0)=(u \bar{x})(i 0): B(i 0)(x / \bar{x}(i 0)) .
$$

And hence again by IH, we obtain (18) and (19).

(2) Let $f: J \rightarrow I$ and $J \Vdash a: A(f, i / 1)$. Then $J, j \Vdash \bar{a}: A(f, i / j)$ as above and we have to show

$$
\begin{aligned}
J \Vdash \operatorname{comp}^{j} B(f, x / \bar{a}, i / j)[\varphi f \mapsto u(f, i / j) \bar{a}]\left(u_{0} f \bar{a}\right) \\
=\operatorname{comp}^{j} B(f, x / \bar{a}, i / j)[\varphi f \mapsto v(f, i / j) \bar{a}]\left(v_{0} f \bar{a}\right): \\
\quad B(f, x / \bar{a}(i 1), i / 1) .
\end{aligned}
$$

But this follows directly from the IH for $J, j \Vdash B(f, x / \bar{a}, i / j)$.

Case SI-C. Let us write $(x: A) \times B$ for the type under consideration. (1) We have

$$
I, i, \varphi \Vdash u .1: A \text { and } I \Vdash u_{0} .1: A[\varphi \mapsto u .1]
$$

so by IH,

$$
I, i \Vdash \text { fill }^{i} A[\varphi \mapsto u .1]\left(u_{0} .1\right): A\left[\varphi \mapsto u .1,(i=0) \mapsto u_{0} .1\right] .
$$

Let us call the above filler $w$. Thus we get $I, i \Vdash B(x / w)$,

$$
I, i, \varphi \Vdash B(x / u .1)=B(x / w) \text { and } I \Vdash B\left(x / u_{0} .1\right)=(B(x / w))(i 0)
$$

and hence

$$
I, i, \varphi \Vdash u .2: B(x / w) \text { and } I \Vdash u_{0} .1:(B(x / w))(i 0)[\varphi \mapsto u .2] .
$$

The IH yields

$$
I \Vdash \operatorname{comp}^{i} B(x / w)[\varphi \mapsto u .2]\left(u_{0} .2\right):(B(x / w))(i 1)[\varphi \mapsto u .2(i 1)] ;
$$

let us write $w^{\prime}$ for the above. By the reduction rules for composition in $\Sigma$-types we get $I \vdash u_{1} \succ_{\mathrm{s}}\left(w(i 1), w^{\prime}\right):(x: A(i 1)) \times B(i 1)$ and hence the Expansion Lemma yields

$$
I \Vdash u_{1}=\left(w(i 1), w^{\prime}\right):(x: A(i 1)) \times B(i 1) .
$$

Which in turn implies the equality

$$
I, \varphi \Vdash u_{1}=\left(w(i 1), w^{\prime}\right)=(u .1(i 1), u .2(i 1))=u(i 1):(x: A(i 1)) \times B(i 1) .
$$

The proof of (2) uses that all notions defining $w$ and $w^{\prime}$ preserve equality (by $\mathrm{IH}$ ), and thus $I \Vdash u_{1} \downarrow=v_{1} \downarrow:(x: A(i 1)) \times B(i 1)$. 

fresh)

Case PA-C. Let us write Path $A a_{0} a_{1}$ for the type under consideration. We obtain (for $j$

$$
\begin{gathered}
I, j \Vdash \operatorname{comp}^{i} A\left[(j=0) \mapsto a_{0},(j=1) \mapsto a_{1}, \varphi \mapsto u j\right]\left(u_{0} j\right): \\
A(i 1)\left[(j=0) \mapsto a_{0}(i 1),(j=1) \mapsto a_{1}(i 1), \varphi \mapsto u(i 1) j\right]
\end{gathered}
$$

by the IH. Using the Expansion Lemma, the reduction rule for composition at Path-types, and Lemma 19 (2) this yields

$$
I \Vdash u_{1} \text { : Path } A(i 1) \tilde{u}(j 0) \tilde{u}(j 1)[\varphi \mapsto\langle j\rangle(u(i 1) j)]
$$

where $\tilde{u}$ is the element in (23) and $u_{1}$ is $\langle j\rangle \tilde{u}$. But $I \Vdash \tilde{u}(j b)=a_{b}(i 1): A(i 1)$, so $I \Vdash u_{1}$ : Path $A(i 1) a_{0}(i 1) a_{1}(i 1)$. Moreover,

$$
I, \varphi \Vdash u_{1}=\langle j\rangle(u(i 1) j)=u(i 1): \text { Path } A(i 1) a_{0}(i 1) a_{1}(i 1)
$$

by the correctness of the $\eta$-rule for paths (Lemma 19 (6)).

Case GL-C. To not confuse with our previous notations, we write $\psi$ for the face formula of $u$, and write $B$ for Glue $[\varphi \mapsto(T, w)] A$.

Thus we are given:

$$
\frac{1 \neq \varphi \in \mathbb{F}(I, i) \quad I, i \Vdash A \quad I, i, \varphi \Vdash w: \text { Equiv } T A \quad I, i, \varphi \Vdash B}{I, i \Vdash B} \text { GL-C }
$$

and also $I, i, \psi \Vdash u: B$ and $I \Vdash u_{0}: B(i 0)[\psi \mapsto u(i 0)]$. Moreover we have $I, i, \varphi \Vdash T$ with shorter derivations by Lemma 23 . We have to show

(i) $I \Vdash u_{1}: B(i 1)$, and

(ii) $I, \psi \Vdash u_{1}=u(i 1): B(i 1)$.

We will be using the Expansion Lemma: let $f: J \rightarrow I$ and consider the reducts of $u_{1} f$ :

$$
u_{1} f \downarrow= \begin{cases}\operatorname{comp}^{j} T f^{\prime}\left[\psi f \mapsto u f^{\prime}\right]\left(u_{0} f\right) & \text { if } \varphi f^{\prime}=1, \\ \operatorname{glue}\left[\varphi(i 1) f \mapsto t_{1} f\right]\left(a_{1} f\right) & \text { otherwise, }\end{cases}
$$

with $f^{\prime}=(f, i / j)$, and $t_{1}$ and $a_{1}$ as in the corresponding reduction rule, i.e.:

$$
\begin{aligned}
a & =\text { unglue }[\varphi \mapsto w] u & & I, i, \psi \\
a_{0} & =\text { unglue }[\varphi(i 0) \mapsto w(i 0)] u_{0} & & I \\
\delta & =\forall i . \varphi & & I \\
a_{1}^{\prime} & =\operatorname{comp}^{i} A[\psi \mapsto a] a_{0} & & I \\
t_{1}^{\prime} & =\operatorname{comp}^{i} T[\psi \mapsto u] u_{0} & & I, \delta \\
\omega & =\operatorname{pres}^{i} w[\psi \mapsto u] u_{0} & & I, \delta \\
\left(t_{1}, \alpha\right) & =\operatorname{equiv} w(i 1)\left[\delta \mapsto\left(t_{1}^{\prime}, \omega\right), \psi \mapsto\left(u(i 1),\langle j\rangle a_{1}^{\prime}\right)\right] a_{1}^{\prime} & & I, \varphi(i 1) \\
a_{1} & =\operatorname{comp}^{j} A(i 1)[\varphi(i 1) \mapsto \alpha j, \psi \mapsto a(i 1)] a_{1}^{\prime} & & I
\end{aligned}
$$

First, we have to check $J \Vdash u_{1} f \downarrow: B(i 1) f$. In case $\varphi f^{\prime}=1$ this immediately follows from the IH. In case $\varphi f^{\prime} \neq 1$, this follows from the IH and the previous lemmas ensuring that notions involved in the definition of $t_{1}$ and $a_{1}$ preserve computability.

Second, we have to check $J \Vdash u_{1} f \downarrow=u_{1} \downarrow f: B(i 1) f$. For this, the only interesting case is when $\varphi f^{\prime}=1$; then we have to check that:

$$
J \Vdash \operatorname{comp}^{j} T f^{\prime}\left[\psi f \mapsto u f^{\prime}\right]\left(u_{0} f\right)=\operatorname{glue}\left[\varphi(i 1) f \mapsto t_{1} f\right]\left(a_{1} f\right): B(i 1) f
$$


Since all the involved notions commute with substitutions, we may (temporarily) assume $f=\mathrm{id}$ and $\varphi=1$ to simplify notation. Then also $\delta=1=\varphi(i 1)$, and hence (using the $\mathrm{IH}$ )

$$
I \Vdash t_{1}=t_{1}^{\prime}=\operatorname{comp}^{i} T[\psi \mapsto u] u_{0}: T(i 1),
$$

so (24) follows from Lemma 22 (5b) and (1).

So the Expansion Lemma yields (i) and $I \Vdash u_{1}=$ glue $\left[\varphi(i 1) \mapsto t_{1}\right] a_{1}: B(i 1)$. (ii) is checked similarly to what is done in [7, Appendix A] using the IH. This proves (1) in this case; for (2) one uses that all notions for giving $a_{1}$ and $t_{1}$ above preserve equality, and thus $I \Vdash u_{1} \downarrow=v_{1} \downarrow: B(i 1)$ entailing $I \Vdash u_{1}=v_{1}: B(i 1)$.

Case U-C. We have

$$
I \vdash \operatorname{comp}^{i} \mathrm{U}[\varphi \mapsto u] u_{0} \succ_{\mathrm{s}} \text { Glue }\left[\varphi \mapsto\left(u(i 1), \operatorname{equiv}^{i} u(i / 1-i)\right)\right] u: \mathrm{U}
$$

thus it is sufficient to prove that the right-hand side is computable, i.e.,

$$
I \Vdash_{1} \text { Glue }\left[\varphi \mapsto\left(u(i 1), \operatorname{equiv}^{i} u(i / 1-i)\right)\right] u_{0}: U
$$

that is,

$$
I \Vdash_{0} \text { Glue }\left[\varphi \mapsto\left(u(i 1), \text { equiv }^{i} u(i / 1-i)\right)\right] u_{0} .
$$

We have $I \Vdash_{0} u_{0}$ so by Lemma 22 (1) it suffices to prove

$$
I \Vdash_{0} \text { equiv }^{i} u(i / 1-i) \text { : Equiv } u(i 1) u_{0} .
$$

To see this recall that the definition of equiv ${ }^{i} u(i / 1-i)$ is defined from compositions and filling operations for types $I, i \Vdash_{0} u$ and $I, i \Vdash_{0} u(i / 1-i)$ using operations we already have shown to preserve computability. But in this case we have as $\mathrm{IH}$, that these composition and filling operations are computable since the derivations of $I, i \Vdash_{0} u$ and $I, i \Vdash_{0} u$ are less complex than the derivation $I \Vdash_{1} \mathrm{U}$ since the level is smaller.

Case NI-C. So we have $J \Vdash A f \downarrow$ for each $f: J \rightarrow I, i$ and $J \Vdash A \downarrow f=A f \downarrow$ (all with a shorter derivation than $I, i \Vdash A$ ). Note that by Lemma 8 (1), we also have $I, i \Vdash A=A \downarrow$.

(1) We have to show $J \Vdash u_{1} f: A(i 1) f \downarrow$ for each $f: J \rightarrow I$. It is enough to show this for $f$ being the identity; we do this using the Expansion Lemma. Let $f: J \rightarrow I$ and $j$ be fresh, $f^{\prime}=(f, i / j)$; we first show $J \Vdash u_{1} f \downarrow: A \downarrow(i 1) f$. We have

$$
J \vdash u_{1} f \succ \operatorname{comp}^{j}\left(A f^{\prime} \downarrow\right)\left[\varphi f \mapsto u f^{\prime}\right] u_{0} f: A f^{\prime}(j 1)
$$

hence also at type $A f^{\prime}(j 1) \downarrow$, and so, by IH (1) for $J, j \Vdash A f^{\prime} \downarrow$, we obtain $J \Vdash u_{1} f \downarrow$ : $A f^{\prime}(j 1) \downarrow$. But $J \Vdash A f^{\prime}(j 1) \downarrow=A \downarrow(i 1) f$, so $J \Vdash u_{1} f \downarrow: A \downarrow(i 1) f$.

Next, we have to show $J \Vdash u_{1} \downarrow f=u_{1} f \downarrow: A \downarrow(i 1) f$. Since $J, j \Vdash A \downarrow f^{\prime}=A f^{\prime} \downarrow$ (with a shorter derivation) we get by IH (3), $J \Vdash u_{1} \downarrow f=u_{1} f \downarrow: A \downarrow f^{\prime}(j 1)$ what we had to show.

Thus we can apply the Expansion Lemma and obtain $I \Vdash u_{1}: A \downarrow(i 1)$ and $I \Vdash u_{1}=$ $u_{1} \downarrow: A \downarrow(i 1)$, and hence also $I \Vdash u_{1}: A(i 1)$ and $I \Vdash u_{1}=u_{1} \downarrow: A(i 1)$. By IH, we also have $I, \varphi \Vdash u_{1}=u_{1} \downarrow=u(i 1): A \downarrow(i 1)=A(i 1)$.

(2) Like above, we obtain

$$
I \Vdash u_{1}=u_{1} \downarrow: A \downarrow(i 1) \text { and } I \Vdash v_{1}=v_{1} \downarrow: A \downarrow(i 1) .
$$

But since the derivation of $I, i \Vdash A \downarrow$ is shorter, and $u_{1} \downarrow=\operatorname{comp}^{i} A \downarrow[\varphi \mapsto u] u_{0}$ and similarly for $v_{1} \downarrow$, the IH yields $I \Vdash u_{1} \downarrow=v_{1} \downarrow: A \downarrow(i 1)$, thus also $I \Vdash u_{1}=v_{1}: A \downarrow(i 1)$, that is, $I \Vdash u_{1}=v_{1}: A(i 1)$ since $I, i \Vdash A=A \downarrow$.

It remains to show that composition preserves forced type equality (i.e., (3) holds). The argument for the different cases is very similar, namely using that the compositions on the 
left-hand and right-hand side of (3) are equal to their respective reducts [by (1)] and then applying the IH for the reducts. We will only present the case NI-E.

Case NI-E. Then $A$ or $B$ is non-introduced and $I, i \Vdash A \downarrow=B \downarrow$ with a shorter derivation. Moreover, by (1) (if the type is non-introduced) or reflexivity (if the type is introduced) we have

$$
\begin{aligned}
& I \Vdash \operatorname{comp}^{i} A[\varphi \mapsto u] u_{0}=\operatorname{comp}^{i}(A \downarrow)[\varphi \mapsto u] u_{0}: A(i 1), \quad \text { and } \\
& I \Vdash \operatorname{comp}^{i} B[\varphi \mapsto u] u_{0}=\operatorname{comp}^{i}(B \downarrow)[\varphi \mapsto u] u_{0}: B(i 1),
\end{aligned}
$$

but the right-hand sides are forced equal by IH.

Lemma 24 The rules for the universe $\bigcup$ are sound:

1. $\Gamma \models A: U \Rightarrow \Gamma \models A$

2. $\Gamma \models A=B: \cup \Rightarrow \Gamma \models A=B$

Moreover, the rules reflecting the type formers in $\mathrm{U}$ are sound.

Proof Of the first two statements let us only prove (2): given $I \Vdash \sigma=\tau: \Gamma$ we get $I \Vdash A \sigma=B \tau: \mathrm{U}$; this must be a derivation of $I \Vdash_{1} A \sigma=B \tau: \mathrm{U}$ and hence we also have $I \Vdash_{0} A \sigma=B \tau$.

The soundness of the rules reflecting the type formers in $U$ is proved very similar to proving the soundness of the type formers. Let us exemplify this by showing soundness for $\Pi$-types in $\mathrm{U}$ : we are give $\Gamma \models A: \mathrm{U}$ and $\Gamma, x: A \models B: \mathrm{U}$, and want to show $\Gamma \models(x: A) \rightarrow B: \mathrm{U}$. Let $I \Vdash \sigma=\tau: \Gamma$, then $I \Vdash A \sigma=A \tau: \cup$, so, as above, $I \Vdash_{0} A \sigma=A \tau$; it is enough to show

$$
J \Vdash_{0} B(\sigma f, x / u)=B(\tau f, x / v)
$$

for $J \Vdash u=v: A \sigma f$ with $f: J \rightarrow I$. Then $J \Vdash(\sigma f, x / u)=(\tau f, x / v): \Gamma, x: A$, hence $J \Vdash B(\sigma f, x / u)=B(\tau f, x / v): \bigcup$ and hence (25).

Proof of Soundness (Theorem 1) By induction on the derivation $\Gamma \vdash \mathscr{J}$.

We have already seen above that most of the rules are sound. Let us now look at the missing rules. Concerning basic type theory, the formation and introduction rules for $\mathrm{N}$ are immediate; its elimination rule and definitional equality follow from the "local" soundness from Lemma 16 as follows. Suppose $\Gamma \models u: \mathrm{N}, \Gamma, x: \mathrm{N} \models C, \Gamma \models z: C(x / 0)$, and $\Gamma \models s:(x: \mathrm{N}) \rightarrow C \rightarrow C(x / \mathrm{S} x)$. For $I \Vdash \sigma=\tau: \mathrm{N}$ we get by Lemma 16 (2)

$$
I \Vdash \text { natrec } u \sigma z \sigma s \sigma=\text { natrec } u \tau z \tau s \tau: C(\sigma, x / u \sigma) .
$$

(Hence $\Gamma \models$ natrec $u z s: C(x / u)$.) Concerning, the definitional equality, if, say, $u$ was of the form $\mathrm{S} v$, then, Lemma 16 (1) gives

$I \Vdash$ natrec $(\mathrm{S} v \sigma) z \sigma s \sigma=\operatorname{natrec}(\mathrm{S} v \tau) z \tau s \tau=(\operatorname{natrec}(\mathrm{S} v \tau) z \tau s \tau) \downarrow: C(\sigma, x / u \sigma)$. and (natrec $(\mathrm{S} v \tau) z \tau s \tau) \downarrow$ is $s \tau v \tau$ (natrec $v \tau z \tau s \tau$ ), proving

$$
\Gamma \models \operatorname{natrec}(\mathrm{S} v) z s=s v(\text { natrec } v z s): C(x / \mathrm{S} v) \text {; }
$$

similarly, the soundness of the other definitional equality is established.

Let us now look at the composition operations: suppose $\Gamma, i: \mathbb{I} \models A, \Gamma \models \varphi: \mathbb{F}, \Gamma, \varphi, i$ : $\mathbb{I} \models u: A$, and $\Gamma \models u_{0}: A(i 0)[\varphi \mapsto u(i 0)]$. Further let $I \Vdash \sigma=\tau: \Gamma$, then for $j$ fresh, $I, j \Vdash \sigma^{\prime}=\tau^{\prime}: \Gamma, i: \mathbb{I}$ where $\sigma^{\prime}=(\sigma, i / j)$ and $\tau^{\prime}=(\tau, i / j)$, hence $I, j \Vdash A \sigma^{\prime}=A \tau^{\prime}$, 
$\varphi \sigma=\varphi \tau, I, j, \varphi \sigma \Vdash u \sigma^{\prime}=u \tau^{\prime}: A \sigma^{\prime}$, and $I \Vdash u_{0} \sigma=u_{0} \tau: A \sigma^{\prime}(j 0)\left[\varphi \sigma \mapsto u \sigma^{\prime}(j 0)\right]$. By Theorem 2,

$$
I \Vdash \operatorname{comp}^{j}\left(A \sigma^{\prime}\right)\left[\varphi \sigma \mapsto u \sigma^{\prime}\right]\left(u_{0} \sigma\right)=\operatorname{comp}^{j}\left(A \tau^{\prime}\right)\left[\varphi \tau \mapsto u \tau^{\prime}\right]\left(u_{0} \tau\right): A \sigma^{\prime}(j 1)
$$

and

$$
I, \varphi \sigma \Vdash \operatorname{comp}^{j}\left(A \sigma^{\prime}\right)\left[\varphi \sigma \mapsto u \sigma^{\prime}\right]\left(u_{0} \sigma\right)=u \sigma^{\prime}(j 1)=u \tau^{\prime}(j 1): A \sigma^{\prime}(j 1)
$$

hence we showed $\Gamma \models \operatorname{comp}^{i} A[\varphi \mapsto u] u_{0}: A(i 1)[\varphi \mapsto u(i 1)]$. Similarly one can justify the congruence rule for composition.

The definitional equalities which hold for comp follow from the second conclusion of Theorem 2 (1), i.e., that a composition is forced equal to its reduct.

The remaining rules for systems follow from their "local" analogues (Lemma 20); let us, say, suppose $\Gamma \models \varphi_{1} \vee \cdots \vee \varphi_{n}=1: \mathbb{F}, \Gamma, \varphi_{i} \models A_{i}$, and $\Gamma, \varphi_{i} \wedge \varphi_{j} \models A_{i}=A_{j}$. For $I \Vdash \sigma=\tau: \Gamma$ we get $k$ with $\varphi_{k} \sigma=\varphi_{k} \tau=1$ like in the proof of Lemma 21 so, writing $A$ for $\left[\varphi_{1} A_{1}, \ldots, \varphi_{n} A_{n}\right]$,

$$
I \Vdash A \sigma=A_{k} \sigma=A_{k} \tau=A \tau
$$

by Lemma 20 and using $\Gamma, \varphi_{k} \models A_{k}$, so $\Gamma \models A$. Likewise, if $\Gamma \models \varphi_{l}=1: \mathbb{F}$ for some $l$, then $I \Vdash A \sigma=A_{l} \sigma=A_{l} \tau$, showing $\Gamma \models A=A_{l}$ in this case. The other rules concerning systems are justified similarly.

The soundness of the remaining rules concerning Glue follow similarly from their "local" version in Lemma 22.

Corollary 1 (Canonicity) If $I$ is a context of the form $i_{1}: \mathbb{I}, \ldots, i_{k}: \mathbb{I}$ and $I \vdash u: \mathrm{N}$, then $I \vdash u=\underline{n}: \mathrm{N}$ for a unique $n \in \mathbb{N}$.

Proof By Soundness, $I \models u$ : N hence $I \Vdash u: \mathrm{N}$, so $I \Vdash u=\underline{n}: \mathrm{N}$ for some $n \in \mathbb{N}$ by Lemma 17, and thus also $I \vdash u=\underline{n}: \mathrm{N}$. The uniqueness follows since $I \vdash \underline{n}=\underline{m}: \mathrm{N}$ yields $I \Vdash \underline{n}=\underline{m}$ : N which is only the case for $n=m$.

Corollary 2 (Consistency) Cubical type theory is consistent, i.e., there is a type in the empty context which is not inhabited.

Proof Consider the type Path $\mathrm{N} 01$ and suppose there is a $u$ with $\vdash u$ : Path $\mathrm{N} 01$. Hence we get $i: \mathbb{I} \vdash u i: \mathrm{N}$, as well as $\vdash u 0=0: \mathrm{N}$ and $\vdash u 1=1: \mathrm{N}$. By Canonicity, we get $n \in \mathbb{N}$ with $i: \mathbb{I} \vdash u i=\underline{n}: \mathrm{N}$, and hence (by substitution) $\vdash u 0=\underline{n}: \mathrm{N}$ and $\vdash u 1=\underline{n}: \mathrm{N}$, so $\vdash 0=1: \mathrm{N}$, contradicting the uniqueness in Corollary 1 .

Remark 5 One could also extend cubical type theory with an empty type $\mathrm{N}_{0}$ whose forcing relation is empty; consistency for this extension is then an immediate consequence of the corresponding Soundness Theorem.

Remark 6 Soundness also implies injectivity of $\Pi$ (and likewise for other type formers) in name contexts: if $I \vdash(x: A) \rightarrow B=\left(x: A^{\prime}\right) \rightarrow B^{\prime}$, then $I \vdash A=A^{\prime}$ and $I, x: A \vdash B=B^{\prime}$. Moreover, we get a canonicity result for the universe $\mathrm{U}$ : if $I \vdash A: \mathrm{U}$, then $A$ is judgmentally equal to an introduced type $B$ with $I \Vdash_{0} B$. 


\section{Extension with Higher Inductive Types}

In this section we discuss two extensions to cubical type theory with two higher inductive types: the circle and propositional truncation. For both extensions it is suitable to generalize path types to dependent path types $\operatorname{Path}^{i} A u v$ where $i$ might now appear in $A$, with $u$ in $A(i 0)$ and $v$ in $A(i 1)$. This extension is straightforward, e.g., the $\beta$-reduction rule for paths now reads

$$
\frac{\Gamma, i: \mathbb{I} \vdash A \quad \Gamma, i: \mathbb{I} \vdash t: A \quad \Gamma \vdash r: \mathbb{I}}{\Gamma \vdash(\langle i\rangle t) r \succ t(i / r): A(i / r)}
$$

and likewise the computability predicates and relations are easily adapted.

\subsection{The Circle}

In this section we sketch how the proof of canonicity can be extended to the system where a circle $S^{1}$ is added; the extension with $n$-spheres is done analogously.

First, we have to extend the reduction relation as follows to incorporate the circle.

$$
\begin{aligned}
& \frac{\Gamma \vdash}{\Gamma \vdash \text { loop } 0 \succ \text { base }: \mathrm{S}^{1}} \\
& \Gamma \vdash \text { loop } 1 \succ \text { base }: \mathrm{S}^{1}
\end{aligned} \quad \frac{\Gamma, i: \mathbb{I} \vdash u: \mathrm{S}^{1}}{\Gamma \vdash \operatorname{comp}^{i} \mathrm{~S}^{1}[1 \mapsto u] u(i 0) \succ u(i 1): \mathrm{S}^{1}}
$$

(For simplicity, we will use comp ${ }^{i} \mathrm{~S}^{1}$ instead of adding yet another constructor hcomp ${ }^{i}$ as was done in in [7].)

Given $\Gamma, x: \mathrm{S}^{1} \vdash C, \Gamma \vdash b: C(x /$ base $)$, and $\Gamma \vdash l:$ Path $^{i} C(x /$ loop $i) b b$ we also add the reduction rules for the elimination

$$
\begin{aligned}
& \Gamma \vdash \mathrm{S}^{1}-\operatorname{elim}_{x . C} \text { base } b l \succ b: C(x / \text { base }) \\
& \Gamma \vdash \mathrm{S}^{1}-\operatorname{elim}_{x . C}(\text { loop } r) b l \succ l r: C(x / \text { loop } r)
\end{aligned}
$$

where $\Gamma \vdash r \neq 1: \mathbb{I}$, and moreover for $\Gamma \vdash \varphi \neq 1: \mathbb{F}$,

$$
\begin{aligned}
& \Gamma \vdash \mathrm{S}^{1}-\operatorname{elim}_{x . C}\left(\operatorname{comp}^{i} \mathrm{~S}^{1}[\varphi \mapsto u] u_{0}\right) b l \\
& \quad \succ \operatorname{comp}^{i} C(x / v)\left[\varphi \mapsto u^{\prime}\right] u_{0}^{\prime}: C\left(x / \operatorname{comp}^{i} \mathrm{~S}^{1}[\varphi \mapsto u] u_{0}\right)
\end{aligned}
$$

where $v=$ fill $^{i} \mathrm{~S}^{1}[\varphi \mapsto u] u_{0}, u^{\prime}=\mathrm{S}^{1}$-elim $_{x . C} u b l, u_{0}^{\prime}=\mathrm{S}^{1}$-elim $x . C u_{0} b l$, and we assumed $i \notin \operatorname{dom} \Gamma$ (otherwise rename $i$ ).

Furthermore, if $\Gamma \vdash t \succ t^{\prime}: \mathrm{S}^{1}$, then

$$
\Gamma \vdash \mathrm{S}^{1}-\operatorname{elim}_{x . C} t b l \succ \mathrm{S}^{1}-\operatorname{elim}_{x . C} t^{\prime} b l: C\left(x / t^{\prime}\right) .
$$

Consequently, we also call expressions introduced if they are of the form $\mathrm{S}^{1}$, base, loop $r$ with $r \notin\{0,1\}$, and comp $\mathrm{S}^{1}[\varphi \mapsto u] u_{0}$ with $\varphi \neq 1$.

Next, the computability predicates and relations are adapted as follows: $I t_{\ell} S^{1}$ and $I \Vdash_{\ell} \mathrm{S}^{1}=\mathrm{S}^{1} . I \Vdash_{\ell} u: \mathrm{S}^{1}$ and $I \Vdash u=v: \mathrm{S}^{1}$ are defined simultaneously (similarly as for $\mathrm{N}$ ): 


$$
\begin{aligned}
& \frac{r \in \mathbb{I}(I)-\{0,1\} \quad I \Vdash_{\ell} \text { loop } 0: \mathrm{S}^{1} \quad I \Vdash_{\ell} \text { loop } 1: \mathrm{S}^{1}}{I \Vdash_{\ell} \text { base }: \mathrm{S}^{1}} \\
& 1 \neq \varphi \in \mathbb{F}(I) \quad I, i, \varphi \Vdash_{\ell} u: \mathrm{S}^{1} \\
& I \Vdash_{\ell} u_{0}: \mathrm{S}^{1} \quad I, \varphi \Vdash_{\ell} u_{0}=u(i 0): \mathrm{S}^{1} \quad I, \varphi \Vdash_{\ell} \operatorname{comp}^{i} \mathrm{~S}^{1}[\varphi \mapsto u] u_{0}: \mathrm{S}^{1} \\
& I \Vdash_{\ell} \operatorname{comp}^{i} \mathrm{~S}^{1}[\varphi \mapsto u] u_{0}: \mathrm{S}^{1}
\end{aligned}
$$$$
u \text { n.i. } \quad \forall f: J \rightarrow I\left(u f !^{S^{1}} \& J \Vdash_{\ell} u f \downarrow^{S^{1}}: S^{1}\right)
$$$$
\frac{\forall f: J \rightarrow I \forall g: K \rightarrow J\left(K \Vdash_{\ell} u f \downarrow g=u f g \downarrow: \mathrm{S}^{1}\right)}{I \Vdash_{\ell} u: \mathrm{S}^{1}}
$$

Note, the (admissible) two last premises in the case for loop are there to not increase the height of the derivation when doing a substitution (Lemma 5); similarly for the last premise in the rule for composition. The relation $I \Vdash_{\ell} u=v: \mathrm{S}^{1}$ is defined analogously, that is, by the usual congruence rules and a clause for when $u$ or $v$ is non-introduced as we have it for $\mathrm{N}$ (see also the next section). To adapt Theorem 2 note that compositions are computable for $\varphi=1$ by using the Expansion Lemma and the reduction rule; using this, compositions are computable by definition also for $\varphi \neq 1$.

\subsection{Propositional Truncation}

We will use a slight simplification of propositional truncation as presented in [7, Section 9.2]. Let us thus recall the typing rules (omitting congruence rules): the formation rule is $\Gamma \vdash\|A\|$ whenever $\Gamma \vdash A$, and likewise $\Gamma \vdash\|A\|: \mathrm{U}$ whenever $\Gamma \vdash A$ : U. Moreover:

$$
\begin{array}{cc}
\frac{\Gamma \vdash a: A}{\Gamma \vdash \operatorname{inc} a:\|A\|} & \frac{\Gamma \vdash u:\|A\| \quad \Gamma \vdash v:\|A\| \quad \Gamma \vdash r: \mathbb{I}}{\Gamma \vdash \text { squash } u v r:\|A\|} \\
\frac{\Gamma \vdash A \quad \Gamma \vdash \varphi: \mathbb{F} \quad \Gamma, \varphi, i: \mathbb{I} \vdash u:\|A\| \quad \Gamma \vdash u_{0}:\|A\|[\varphi \mapsto u(i / 0)]}{\Gamma \vdash \operatorname{hcomp}_{\|A\|}^{i}[\varphi \mapsto u] u_{0}:\|A\|}
\end{array}
$$

with the judgmental equalities (omitting context and type):

$$
\text { squash } u v 0=u \quad \text { squash } u v 1=v \quad \operatorname{hcomp}_{\|A\|}^{i}\left[1_{\mathbb{F}} \mapsto u\right] u_{0}=u(i / 1)
$$

Note that the type in hcomp ${ }^{i}$ does not depend on $i$ and we call these homogeneous compositions. The eliminator, given $\Gamma \vdash A$ and $\Gamma, z:\|A\| \vdash C(z)$, is given by the rule

$$
\begin{gathered}
\Gamma \vdash w:\|A\| \quad \Gamma \vdash t:(a: A) \rightarrow C(\text { inc } a) \\
\Gamma \vdash p:(u v:\|A\|)(x: C(u))(y: C(v)) \rightarrow \operatorname{Path}^{i}(C(\text { squash } u v i)) x y \\
\Gamma \vdash \operatorname{elim}_{z . C} w t p: C(w)
\end{gathered}
$$

together with judgmental equalities (assuming $i$ fresh):

$$
\begin{aligned}
& \operatorname{elim}_{z . C}(\text { inc } a) t p=t a \\
& \operatorname{elim}_{z . C}(\text { squash } u v r) t p=p u v\left(\operatorname{elim}_{z . C} u t p\right)\left(\operatorname{elim}_{z . C} v t p\right) r
\end{aligned}
$$

$\operatorname{elim}_{z . C}\left(\operatorname{hcomp}^{i}[\varphi \mapsto u] u_{0}\right) t p=\operatorname{comp}^{i} C(z / w)\left[\varphi \mapsto \operatorname{elim}_{z . C} u t p\right]\left(\operatorname{elim}_{z . C} u_{0} t p\right)$

where $w=\operatorname{hcomp}^{j}\left[\varphi \mapsto u(i / i \wedge j),(i=0) \mapsto u_{0}\right] u_{0}$. 
Instead of transp and squeeze in [7] we take the following forward operation:

$$
\frac{\Gamma, i: \mathbb{I} \vdash A \quad \Gamma \vdash r: \mathbb{I} \quad \Gamma \vdash u:\|A(i / r)\|}{\Gamma \vdash \mathrm{fwd}_{i . A} r u:\|A(i / 1)\|}
$$

which comes with the judgmental equalities:

$$
\begin{gathered}
\text { fwd } 1 u=u \\
\text { fwd } r(\operatorname{inc} a)=\operatorname{inc}\left(\operatorname{comp}^{i} A(i / i \vee r)[(r=1) \mapsto a] a\right) \\
\text { fwd } \left.r(\operatorname{squash} u v s)=\operatorname{squash}_{(\mathrm{fwd} r} u\right)(\mathrm{fwd} r v) s \\
\text { fwd } r\left(\operatorname{hcomp}_{\|A(i / r)\|}^{j}[\varphi \mapsto u] u_{0}\right)=\operatorname{hcomp}_{\|A(i / 1)\|}^{j}[\varphi \mapsto \text { fwd } r u]\left(\text { fwd } r u_{0}\right)
\end{gathered}
$$

Composition for $\|A\|$ is now explained using fwd and homogeneous composition:

$$
\operatorname{comp}^{i}\|A\|[\varphi \mapsto u] u_{0}=\operatorname{hcomp}_{\|A(i / 1)\|}^{i}\left[\varphi \mapsto \operatorname{fwd}_{j . A(i / j)} i u\right]\left(\operatorname{fwd}_{i . A} 0 u_{0}\right)
$$

Next, we extend the reduction relation by directing the above judgmental equalities from left to right, but requiring the following extra conditions to guarantee determinism (additionally to the suppressed well-typedness). The directed versions of (26)-(28) require $r \neq 1$; (27) and (28) additionally require $s \neq 1$ and $\varphi \neq 1$, respectively. Similarly for the reductions of elim. Additionally, we need congruence rules:

$$
\frac{\Gamma, i: \mathbb{I} \vdash A \quad \Gamma \vdash r \neq 1: \mathbb{F} \quad \Gamma \vdash u \succ v:\|A(i / r)\|}{\Gamma \vdash \mathrm{fwd}_{i . A} r u \succ \mathrm{fwd}_{i . A} r v:\|A(i / 1)\|}
$$

and a similar such rule for elim. Correspondingly, we also call expressions of the following form introduced: $\|A\|$, inc $a$, squash $u v r$ with $r \neq 1$, and hcomp's with $\varphi \neq 1$.

To incorporate propositional truncation in the computability predicates we add new the formation rules:

$$
\frac{I, 1 \Vdash_{\ell} A}{I \Vdash_{\ell}\|A\|} \text { РT-C } \quad \frac{I \Vdash_{\ell} A=B}{I \Vdash_{\ell}\|A\|=\|B\|} \text { PT-E }
$$

And in the case $I \Vdash_{\ell} A$ was derived via PT-C the definition of $I \Vdash_{\ell} u: A$ is extended to:

$$
\begin{aligned}
& \frac{I \Vdash_{\ell} a: A}{I \Vdash_{\ell} \operatorname{inc} a:\|A\|} \\
& \frac{0,1 \neq r \in \mathbb{I}(I) \quad I \Vdash_{\ell} \text { squash } u v 0:\|A\| \quad I \Vdash_{\ell} \text { squash } u v 1:\|A\|}{I \Vdash_{\ell} \text { squash } u v r:\|A\|} \\
& 1 \neq \varphi \in \mathbb{F}(I) \quad I, i, \varphi \Vdash_{\ell} u:\|A\| \\
& \frac{I \Vdash_{\ell} u_{0}:\|A\| \quad I, \varphi \Vdash_{\ell} u_{0}=u(i 0):\|A\| \quad I, \varphi \Vdash_{\ell} \operatorname{hcomp}_{\|A\|}^{i}[\varphi \mapsto u] u_{0}:\|A\|}{I \Vdash_{\ell} \operatorname{hcomp}_{\|A\|}^{i}[\varphi \mapsto u] u_{0}:\|A\|} \\
& u \text { n.i. } \quad \forall f: J \rightarrow I\left(u f !^{\|A f\|} \& J \Vdash_{\ell} u f \downarrow\|A f\|:\|A f\|\right) \\
& \forall f: J \rightarrow I \forall g: K \rightarrow J\left(K \Vdash_{\ell} u f \downarrow g=u f g \downarrow:\|A f g\|\right) \\
& I \Vdash_{\ell} u:\|A\|
\end{aligned}
$$


As before, the rather unnatural formulation of the rules for squash and hcomp is to ensure that the height of a derivation is not increased after performing a substitution (Lemma 5).

$$
\begin{aligned}
& \frac{I \Vdash_{\ell} a=a^{\prime}: A}{I \Vdash_{\ell} \operatorname{inc} a=\operatorname{inc} a^{\prime}:\|A\|} \quad \frac{0,1 \neq r \in \mathbb{I}(I) \quad I \Vdash_{\ell} u=u^{\prime}:\|A\| \quad I \Vdash_{\ell} v=v^{\prime}:\|A\|}{I \Vdash_{\ell} \operatorname{squash} u v r=\operatorname{squash} u^{\prime} v^{\prime} r:\|A\|} \\
& 1 \neq \varphi \in \mathbb{F}(I) \quad I, i, \varphi \Vdash_{\ell} u=u^{\prime}:\|A\| \\
& I \Vdash_{\ell} u_{0}=u_{0}^{\prime}:\|A\| \quad I, \varphi \Vdash_{\ell} \operatorname{hcomp}_{\|A\|}^{i}[\varphi \mapsto u] u_{0}=\operatorname{hcomp}_{\|A\|}^{i}\left[\varphi \mapsto u^{\prime}\right] u_{0}^{\prime}:\|A\| \\
& I \Vdash_{\ell} \operatorname{hcomp}_{\|A\|}^{i}[\varphi \mapsto u] u_{0}=\operatorname{hcomp}_{\|A\|}^{i}\left[\varphi \mapsto u^{\prime}\right] u_{0}^{\prime}:\|A\| \\
& \frac{u \text { or } u^{\prime} \text { n.i. } \quad \forall f: J \rightarrow I\left(J \Vdash_{\ell} u f \downarrow^{\|A f\|}=u^{\prime} f \downarrow^{\|A f\|}:\|A f\|\right)}{I \Vdash_{\ell} u=u^{\prime}:\|A\|}
\end{aligned}
$$

We now sketch how one can extend the proofs of Sects. 3 and 4. The additional case in the Expansion Lemma is handled as for natural numbers. Next, one proves the introduction rules for inc, squash, and hcomp correct. To handle the new case PT-C for propositional truncation in Theorem 2 one has to simultaneously prove

$$
\begin{aligned}
& I \Vdash u:\|A(i / r)\| \Rightarrow I \Vdash \operatorname{fwd} r u:\|A(i / 1)\| \\
& I \Vdash u=v:\|A(i / r)\| \Rightarrow I \Vdash \mathrm{fwd} r u=\mathrm{fwd} r v:\|A(i / 1)\|
\end{aligned}
$$

by a side induction on the premises. Finally, one can then show soundness of elim.

We not only get the corresponding canonicity result for the extended theory, but we can also extract witnesses from $\|A\|$ as long as we are in a name context:

Theorem 3 If $I \vdash A$ and $I \vdash u:\|A\|$, then $I \vdash v:$ A for some $v$, where $I$ is a context of the form $i_{1}: \mathbb{I}, \ldots, i_{n}: \mathbb{I}$ with $n \geq 0$.

Proof By Soundness we get $I \models A$ and $I \models u:\|A\|$, and hence also $I \Vdash A$ and $I \Vdash u:\|A\|$. By induction on $I \Vdash u:\|A\|$ we show that there is some $v$ such that $I \Vdash v: A$. In the case for inc this is direct; any other case follows from the IH. Thus also $I \vdash v: A$ as required.

As a direct consequence we get that the logic of mere propositions (cf. [14, Section 3.7]) of cubical type theory satisfies the following existence property. Define $\exists(x: A) B$ as the truncated $\Sigma$-type, i.e., $\|(x: A) \times B\|$.

Corollary 3 If $I \vdash \exists(x: A) B(x)$ is true (i.e., there is a term inhabiting the type), then there exists $u$ with $I \vdash u:$ A such that $I \vdash B(x / u)$ is true, where $I$ is a context of the form $i_{1}: \mathbb{I}, \ldots, i_{n}: \mathbb{I}$ with $n \geq 0$.

\section{Conclusion}

We have shown canonicity for cubical type theory [7] and its extensions with the circle and propositional truncation. This establishes that the judgmental equalities of the theory are sufficient to compute closed naturals to numerals; indeed, we have even given a deterministic reduction relation to do so. It should be noted that we could have also worked with the corresponding untyped reduction relation $A \succ B$ and then take $I \vdash A \succ B$ to mean $I \vdash A=B, I \vdash A, I \vdash B$, and $A \succ B$ etc.

To prove canonicity we devised computability predicates (and relations) which, from a set-theoretic perspective, are constructed using the least fixpoint of a suitable operator. It 
is unlikely that this result is optimal in terms of proof-theoretic strength; we conjecture that it is possible to modify the argument to only require the existence of a fixpoint of a suitably modified operator (and not necessarily its least fixpoint); this should be related to how canonicity is established in [3].

We expect that the present work can be extended to get a normalization theorem and to establish decidability of type checking for cubical type theory (and proving its implementation $^{2}$ correct). One new aspect of such an adaption is to generalize the computability predicates and relations to expressions in any contexts in which we get new introduced expressions given by systems; moreover, we will have to consider reductions in such general contexts as well which has to ensure that, say, variables of path-types compute to the right endpoints.

Another direction of future research is to investigate canonicity of various extensions of cubical type theory, especially adding resizing rules.

Acknowledgements I thank Carlo Angiuli, Thierry Coquand, Robert Harper, and Bassel Mannaa for discussions about this work, as well as Milly Maietti who also suggested to investigate the existence property. I am also grateful for the comments by the anonymous reviewer.

Open Access This article is distributed under the terms of the Creative Commons Attribution 4.0 International License (http://creativecommons.org/licenses/by/4.0/), which permits unrestricted use, distribution, and reproduction in any medium, provided you give appropriate credit to the original author(s) and the source, provide a link to the Creative Commons license, and indicate if changes were made.

\section{References}

1. Abel, A., Coquand, T., Mannaa, B.: On the decidability of conversion in type theory (2016). (Abstract for TYPES (2016))

2. Abel, A., Scherer, G.: On irrelevance and algorithmic equality in predicative type theory. Log. Methods Comput. Sci. 8(1), 1-36 (2012). (TYPES'10 special issue)

3. Angiuli, C., Harper, R.: Computational higher type theory II: dependent cubical realizability (2016). Preprint arXiv:1606.09638v1 [cs.LO]

4. Angiuli, C., Harper, R., Wilson, T.: Computational higher type theory I: abstract cubical realizability (2016). Preprint arXiv:1604.08873v1 [cs.LO]

5. Angiuli, C., Harper, R., Wilson, T.: Computational higher-dimensional type theory. In: Proceedings of the 44th ACM SIGPLAN Symposium on Principles of Programming Languages, POPL 2017, pp. 680-693. ACM, New York, NY, USA (2017). https://doi.org/10.1145/3009837.3009861

6. Bezem, M., Coquand, T., Huber, S.: A model of type theory in cubical sets. In: Matthes, R., Schubert, A. (eds.) 19th International Conference on Types for Proofs and Programs (TYPES 2013), Leibniz International Proceedings in Informatics (LIPIcs), vol. 26, pp. 107-128. Schloss Dagstuhl-Leibniz-Zentrum für Informatik, Dagstuhl (2014). https://doi.org/10.4230/LIPIcs.TYPES.2013.107

7. Cohen, C., Coquand, T., Huber, S., Mörtberg, A.: Cubical type theory: a constructive interpretation of the univalence axiom. In: Uustalu, T. (ed.) 21st International Conference on Types for Proofs and Programs (TYPES 2015). Leibniz International Proceedings in Informatics (LIPIcs), vol. 69, pp. 134. Schloss Dagstuhl-Leibniz-Zentrum für Informatik, Dagstuhl (2018). https://doi.org/10.4230/LIPIcs. TYPES.2015.5

8. Coquand, T., Mannaa, B.: The independence of Markov's principle in type theory. In: Kesner, D., Pientka, B. (eds.) 1st International Conference on Formal Structures for Computation and Deduction (FSCD 2016), Leibniz International Proceedings in Informatics (LIPIcs), vol. 52, pp. 17:1-17:18. Schloss DagstuhlLeibniz-Zentrum für Informatik, Dagstuhl (2016). https://doi.org/10.4230/LIPIcs.FSCD.2016.17

9. Dybjer, P.: A general formulation of simultaneous inductive-recursive definitions in type theory. J. Symb. Log. 65(2), 525-549 (2000)

10. Huber, S.: A model of type theory in cubical sets. Licentiate thesis, University of Gothenburg (2015)

11. Huber, S.: Cubical intepretations of type theory. Ph.D. thesis, University of Gothenburg (2016)

2 Available at https://github.com/mortberg/cubicaltt. 
12. Martin-Löf, P.: An intuitionistic theory of types. In: Sambin, G., Smith, J.M. (eds.) Twenty-Five Years of Constructive Type Theory (Venice, 1995), Oxford Logic Guides, vol. 36, pp. 127-172. Oxford University Press, Oxford (1998)

13. Tait, W.W.: Intensional interpretations of functionals of finite type I. J. Symb. Log. 32(2), 198-212 (1967)

14. The Univalent Foundations Program: Homotopy Type Theory: Univalent Foundations of Mathematics. Institute for Advanced Study. http://homotopytypetheory.org/book (2013)

15. Voevodsky, V.: The equivalence axiom and univalent models of type theory. (Talk at CMU on February 4, 2010) (2014). Preprint arXiv:1402.5556 [math.LO] 